Pacific Journal of Mathematics

ON EQUIVALENCES OF BRANCHED COVERINGS AND THEIR 


\title{
ON EQUIVALENCES OF BRANCHED COVERINGS AND THEIR ACTION ON HOMOLOGY
}

\author{
WiLliam KaZEZ
}

This paper studies equivalences of stable simple branched coverings of surfaces. We give necessary and sufficient conditions for a pair of homeomorphisms $f$ and $g$ of surfaces $M$ and $N$ respectively to be homologous to homeomorphisms $\bar{f}$ and $\bar{g}$ which form an equivalence of two prespecified stable simple branched covers $\psi_{1}$ and $\psi_{2}$. That is, homeomorphisms $\bar{f}$ and $\tilde{g}$ such that

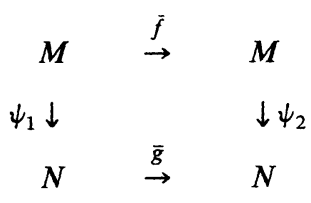

commutes are shown to exist if and only if $\psi_{2 *} f_{*}=g_{*} \psi_{1 *}$ from $H_{*}(M)$ to $H_{*}(N)$.

The proof relies on a uniqueness theorem of Hamilton and Berstein, Edmonds to restate the problem in terms of self equivalences of certain simple branched covers. Many equivalences of branched covers are constructed, and it is shown that the action on homology of these equivalences generates an appropriate subgroup of the symplectic group.

\section{Chapter 1}

Preliminaries. All spaces and maps will be piecewise linear. $M$ and $N$ will denote orientable surfaces while $M_{g}$ and $N_{g}$ will denote closed orientable surfaces of genus $g$. Maps will be orientation preserving unless otherwise specified.

A branched cover is a finite to one open map $\varphi: M \rightarrow N$. In addition, we shall assume that branched covers are primitive, that is induce surjections on the fundamental group. The degree of $\varphi$ is $\max _{x \in N} \#\left\{\varphi^{-1}(x)\right\}$. The singular set of $\varphi$ is the (finite) set of points in $M$ near which $\varphi$ fails to be a local homeomorphism. The branch set $B$ of $\varphi$ is the image under $\varphi$ of the singular set. A branched cover is simple if for all $x \in N, \#\left\{\varphi^{-1}(x)\right\}$ $\in\{d, d-1\}$. Equivalently, a branched cover is simple if each branch point is covered by exactly one singular point, and near this point the map is of degree 2 . 


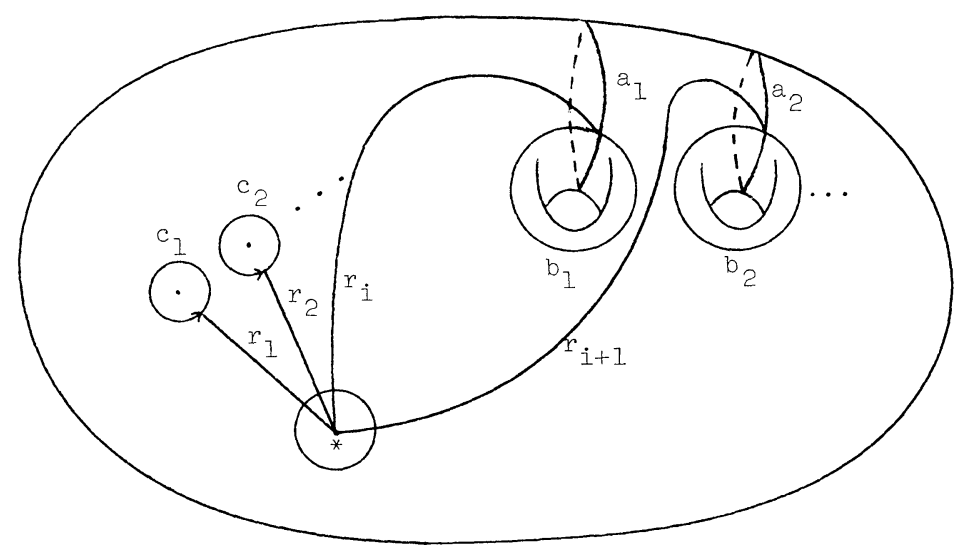

Diagram 1.1

Two branched covers $\varphi$ and $\psi$ from $M$ to $N$ are equivalent if there exist homeomorphisms $f$ of $M$ and $g$ of $N$ such that

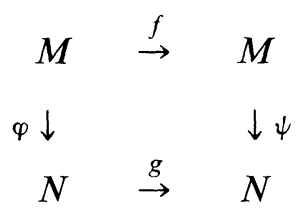

commutes. $\varphi$ is determined up to equivalence by the associated unbranched cover $\varphi \mid: M-\varphi^{-1}(B) \rightarrow N-B$.

From ordinary covering space theory the associated unbranched cover may be studied by picking a base point $* \in N-B$, labeling $\varphi^{-1}(*)$, $1,2, \ldots, d$, and then looking at the representation $\rho(\varphi): \pi_{1}(N-B, *) \rightarrow$ $S(d)$ where $\rho(\varphi)$ is the action of the fundamental group on a fibre and $S(d)$ is the symmetric group on $d$ letters. To study $\rho(\varphi)$ we first choose generators of $\pi_{1}(N-B, *)$ as follows. Let $a_{t}, b_{i}$ be embedded $S^{1}$ 's representing the standard generators of $H_{1}(N)$ such that $a_{i} \cap a_{j}=b_{i} \cap b_{j}$ $=a_{i} \cap b_{j}=\varnothing$ for all $i \neq j$ and $a_{i} \cap b_{t}=$ pt. Choose small $S^{1}$ 's $c_{l}$ about each branch pt. Next choose arcs $r_{i}$ from $*$ to a point on each $c_{l}$ and choose more arcs $r_{i}$ from $*$ to each $a_{i} \cap b_{i}$. Different arcs $r_{i}$ should meet only at $*$ and they should meet a small circle about $*$ in the order shown in Diagram 1.1.

These arcs $r_{l}$ together with the curves $c_{1}, c_{2}, \ldots ; a_{1}, b_{1}, a_{2}, b_{2}, \ldots$ form an ordered set of generators for $\pi_{1}(N-B, *)$. Applying $\rho(\varphi)$ to these generators gives an ordered set called a Hurwitz system $[\mathbf{H u}]$ of the branched cover $\varphi$.

It is shown in [BE2] that if $\phi$ is a simple stable branched cover (that is genus $M \geq d \cdot$ genus $N$ ) there then exists a different choice of arcs $r_{l}$ and 
curves $a_{i}$ and $b_{i}$ together with a relabeling of $\phi^{-1}(*)$ which yield a particularly simple Hurwitz system. That is they prove

(1.1) Stable Canonical Form Theorem: Every stable simple branched covering $\phi: M \rightarrow N$ of degree d has Hurwitz system (12),..,,(12), (12), (13), (13),...,(1d), (1d); (1),..,(1). From this follows

(1.2) Stable UniQueness Theorem: Simple branched covers $\phi_{1}$ and $\phi_{2}$ from $M$ to $N$ both with the same degree $d$ in the stable range are equivalent.

This theorem was proved by Hamilton in [Ha] and in greater generality than stated above by Berstein and Edmonds in [BE2].

If $\varphi: M \rightarrow N$ is a simple branched cover of degree $d$ we say an arc $\beta \subseteq N$ is of type $(i j),(i j)$ if $* \in \hat{\beta}, \hat{\beta} \cap B=\varnothing, \#\{\beta \cap B\}=2$, and $\varphi^{-1}(\beta)$ consists of $d-2$ arcs together with one loop $\alpha$ such that $\alpha \cap$ $\varphi^{-1}(*)=\{i, j\}$. The inverse image of a small regular neighborhood of $\beta$ consists of $d-2$ disks mapped homeomorphically by $\phi$ and one annulus mapped with degree 2 by $\phi$. It follows from [Hil; §2], [BE 1] that $T(\beta)$, the arc twist about $\beta$, is covered by $T(\alpha)$, the loop twist about $\alpha$ (see [Li1], [Li2], [Bir; p. 166]), together with $d-2$ arc twists. We now have the commutative diagram

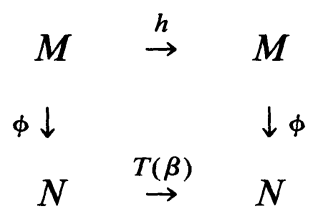

(1.3) LeMMA. Let $\varphi: M \rightarrow N$ be a simple branched cover with degree in the stable range. Then for all $i, j$ with $1 \leq i<j \leq d$ there exist homeomorphism $h, \bar{h}$ each isotopic to the identity such that

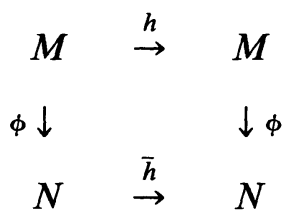

commutes, $\bar{h}(*)=*$, and $h \mid \phi^{-1}(*)$ is the permutation $(i j)$.

Proof. We may assume that $\phi$ has the Hurwitz system (12),..,(12), (13), (13), ..,(1d), (1d); (1),..,(1). Let $\beta_{1}$ be an arc in $N$ of type (ij), (ij). Then $T\left(\beta_{1}\right)$ lifts to a homeomorphism $h_{1}$ inducing the desired permutation $(i j)$ on $\phi^{-1}(*)$. Choose another arc $\beta_{2}$ not containing $*$ but 
differing from $\beta_{1}$ only in a small neighborhood of $*$. Then $T\left(\beta_{2}\right)$ lifts to a homeomorphism $h_{2}$ isotopic to $h_{1}$ which is the identity map on $\phi^{-1}(*)$. It follows that $h=h_{2}^{-1} h_{1}$ and $\bar{h}=T\left(\beta_{2}\right)^{-1} T\left(\beta_{1}\right)$ have the desired properties.

We shall use the following definitions in the next chapter.

Let $\varphi$ and $\varphi^{\prime}$ be simple branched covers of degree $d$ from $M$ to $N$ and $M^{\prime}$ to $N^{\prime}$ respectively. Choose disks $D, D^{\prime}$ with $* \in \partial D \subseteq D \subseteq N-B$ and $*^{\prime} \in \partial D^{\prime} \subseteq D^{\prime} \subseteq N^{\prime}-B^{\prime}$. We may then form a new branched cover

$$
\varphi \# \varphi^{\prime}:\left(M-\varphi^{-1}(\stackrel{\circ}{D})\right) \#\left(M^{\prime}-\varphi^{\prime-1}\left(\stackrel{\circ}{D}^{\prime}\right)\right) \rightarrow(N-\stackrel{\circ}{D}) \#\left(N^{\prime}-\stackrel{\circ}{D}^{\prime}\right) \text {, }
$$

where in the first connected sum we identify for each $i, 1 \leq i \leq d$, the component of $\varphi^{-1}(\partial D)$ containing $i \in \varphi^{-1}(*)$ with the component of $\varphi^{\prime-1}\left(\partial D^{\prime}\right)$ containing $i \in \varphi^{\prime-1}\left(*^{\prime}\right)$, and in the second connected sum we identify $\partial D$ with $\partial D^{\prime}$. This is the fibre connected sum of $\varphi$ and $\varphi^{\prime}$.

If in the above definition $D$ and $D^{\prime}$ are instead chosen to contain exactly one branch point and $\rho(\varphi)[\partial D]=\rho\left(\varphi^{\prime}\right)\left[\partial D^{\prime}\right] \in S(d)$ we may repeat the construction (though in this case there are now only $d-1$ components of $\varphi^{-1}(\partial D)$ and $\left.\varphi^{\prime-1}\left(\partial D^{\prime}\right)\right)$. This final branched cover is called the connected sum along a singular fibre of $\varphi$ and $\varphi^{\prime}$.

\section{CHAPTER 2}

Examples of Branched Covers and their Equivalences. In this chapter, we shall construct examples of simple branched covers and examples of equivalences of these branched covers, i.e. homeomorphisms $h, \bar{h}$ such that

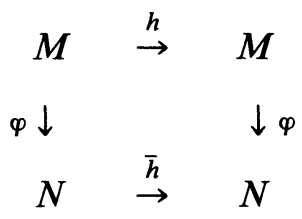

commutes. In (2.1), (2.2) and (2.3) all equivalences constructed will satisfy $\bar{h} \simeq \mathrm{id}_{N}$.

(2.1) Let $N=S^{1} \times S^{1}$, let $M_{d}$ be a surface of genus $d$ with $d \geq 3$, and let $\varphi$ be a simple branched cover of degree $d$. By (1.2) we may assume that $\varphi$ is the connected sum along a fibre of a degree $d$ simple branched cover $\varphi_{1}: S^{2} \rightarrow S^{2}$ with the trivial cover $\varphi_{2}$ from $d$ copies of $S^{1} \times S^{1}$ to $S^{1} \times S^{1}$. See Diagram 2.1.

Choose loops $a^{i}, b^{i}$ and $a, b$ which represent generators of $H_{1}(M)$ and $H_{1}(N)$ respectively such that $\varphi\left(a^{i}\right)=a, \varphi\left(b^{i}\right)=b$ for $i=1, \ldots, d$.

We now give three types of equivalences of this branched cover. 

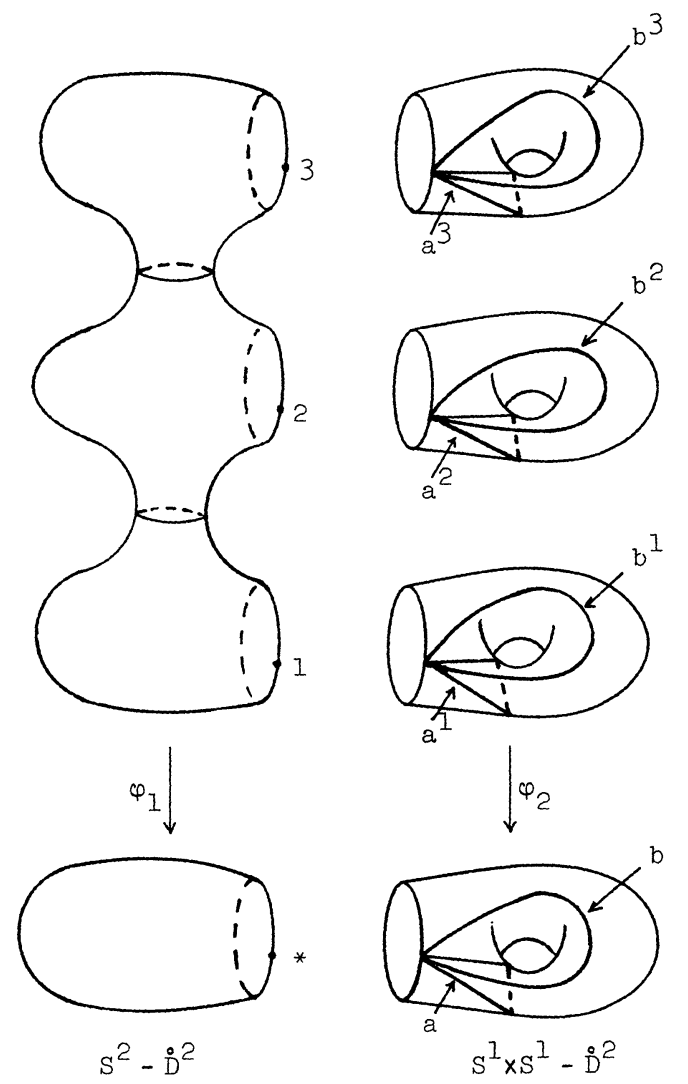

$$
\varphi: M_{3} \rightarrow \dot{S}^{I} \times S^{I}
$$

\section{DIAGRAM 2.1}

(2.1.1) By Lemma (1.3) there is an equivalence of $\varphi_{1}$ which permutes the $i$ and $j$ elements of $\varphi_{1}^{-1}\left(*_{1}\right)$. We may assume that this equivalence fixes a disk $\stackrel{\circ}{D}^{2} \subseteq S^{2}$ with $*_{1} \in \partial D^{2}$. Let $P^{i j}: M_{d} \rightarrow M_{d}$ be the homeomorphism obtained by taking the connected sum along the fibre of the above equivalence of $\varphi_{1}$ and the equivalence of $\varphi_{2}$ which permutes the $i$ th and $j$ th copies of $S^{1} \times S^{1}$.

(2.1.2) As in Lemma (1.3), we may choose an $\operatorname{arc} \beta$ of type (ij), (ij) in $S^{2}-\check{D}^{2}$. The inverse image by $\varphi$ in $M_{d}$ of the connected sum of $\beta$ and the loop $a$ is the disjoint union of $d-2$ arcs and 1 loop which is homologous to $\left[a^{i}\right]-\left[a^{j}\right] \in H_{1}\left(M_{d}\right)$. Denote by $T\left(\left[a^{i}\right]-\left[a^{j}\right]\right)$ the equivalence which covers the arc twist $T(\beta \# a)$.

We shall be studying the effect on $H_{1}\left(M_{d}\right)$ of these equivalences. It should be noted that if $c$ is a curve representing a homology class in $H_{1}\left(M_{d}\right)$ then the twist homeomorphism $T([c])$ is not well defined, only its action on $H_{1}(M)$ is well defined. Details are contained in Chapter 3. 

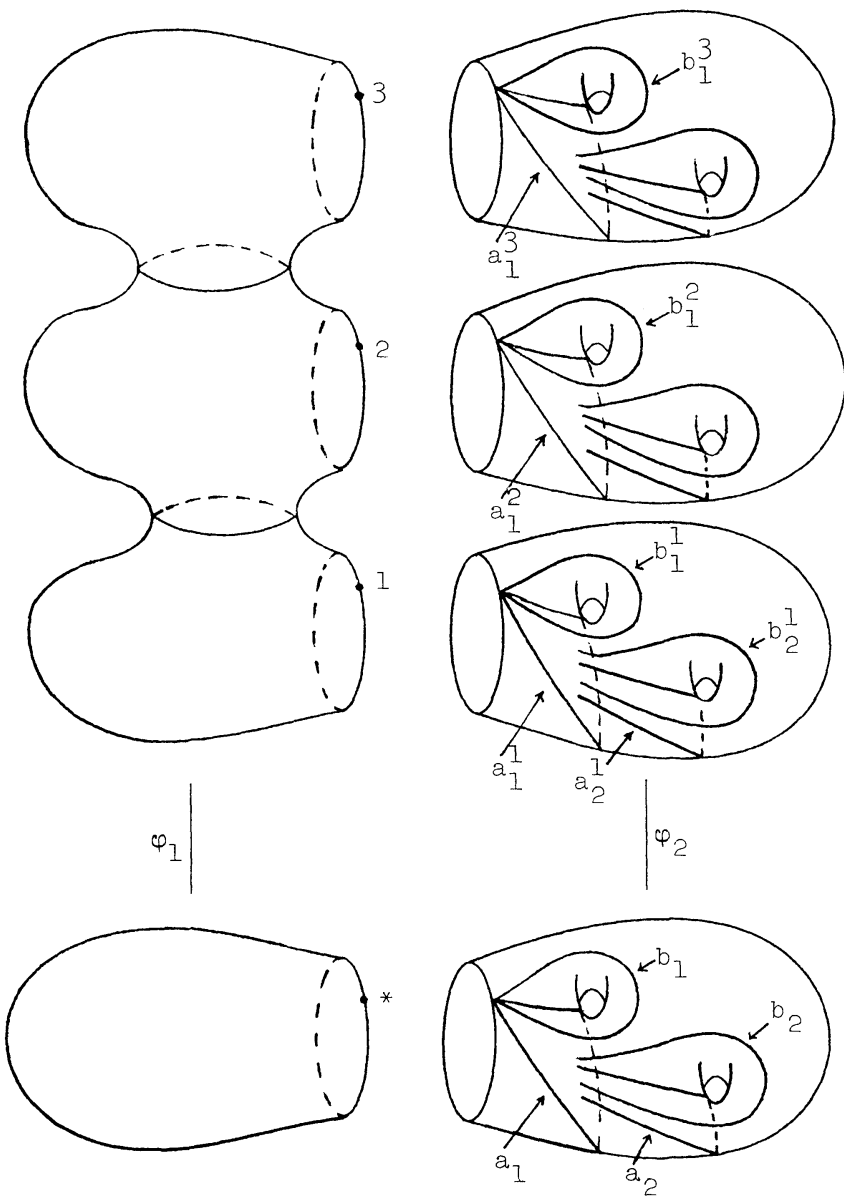

$S^{2}-\dot{D}^{2}$

$$
\mathrm{N}_{2}-\dot{D}^{2}
$$

$\varphi: \mathrm{M}_{3 \cdot 2} \rightarrow \mathrm{N}_{2}$

\section{Diagram 2.2}

(2.1.3) If $a, a^{i}, a^{j}$ are replaced by $b, b^{i}, b^{J}$ in (2.1.2), it follows that $T\left(\left[b^{i}\right]-\left[b^{j}\right]\right)$ is also an equivalence of $\varphi$.

(2.2) Let $\varphi$ be a simple branched cover of degree $d \geq 3$ from $M_{k d} \rightarrow N_{k}$ where $k>1$. By (1.2) we may assume that $\varphi$ is the fibre connected sum of a degree $d$ simple branched cover $\varphi_{1}: S^{2} \rightarrow S^{2}$ with the trivial cover $\varphi_{2}$ from $k$ disjoint copies of $N_{k}$ to $N_{k}$. See Diagram 2.2. Choose loops $a_{s}^{i}, b_{s}^{i}$ and $a_{s}, b_{s}(i=1,2, \ldots, d$ and $s=1,2, \ldots, k)$ representing the standard generators of $H_{1}\left(M_{k d}\right)$ and $H_{1}\left(N_{k}\right)$ respectively which satisfy $\varphi\left(a_{s}^{i}\right)=a_{s}$, $\varphi\left(b_{s}^{i}\right)=b_{s}$.

We have the following examples of equivalences of $\varphi: M_{k d} \rightarrow N_{k}$. 
(2.2.1) Regard $N_{k}$ as $S^{2} \#\left(S^{1} \times S^{1}\right) \# \cdots \#\left(S^{1} \times S^{1}\right)$ and apply the construction of (2.1.1) to the $i$ th $S^{1} \times S^{1}$. This gives an equivalence $P_{t}^{i j}$ which permutes the $i$ and $j$ tori in $M_{k d}$ above the $t$ th copy of $S^{1} \times S^{1}$.

(2.2.2) Similarly (2.1.2) applied to the $t$ th torus in $N_{k}$ shows that the twists $T\left(\left[a_{t}^{i}\right]-\left[a_{t}^{j}\right]\right)$ and $T\left(\left[b_{t}^{i}\right]-\left[b_{t}^{j}\right]\right)$ are equivalences.

(2.2.3) Regard $\varphi$ as $\varphi_{1} \# \varphi_{2}$ and $N_{k}$ as $S^{2} \# N_{k}$. Choose an arc $\beta$ in $S^{2}-\check{D}^{2}$ of type $(i j),(i j)$ and a loop $c$ in $N_{k}-\check{D}^{2}$ homologous to $\left[a_{s}\right]+\left[a_{t}\right](s \neq t)$. The connected sum of these is an arc in $S^{2} \# N_{k}$ of type $(i j),(i j)$ covered by a loop homologous to $\left[a_{s}^{i}\right]-\left[a_{s}^{j}\right]+\left[a_{t}^{i}\right]-\left[a_{t}^{j}\right]$. This shows that $T\left(\left[a_{s}^{i}\right]-\left[a_{s}^{j}\right]+\left[a_{t}^{i}\right]-\left[a_{t}^{j}\right]\right)$ is an equivalence of $\varphi$.

(2.2.4) Repeating (2.2.3) but choosing the loop $c$ to be homologous to $\left[a_{s}\right]+\left[b_{t}\right]$ shows that $T\left(\left[a_{s}^{i}\right]-\left[a_{s}^{j}\right]+\left[b_{t}^{i}\right]-\left[b_{t}^{j}\right]\right)$ is an equivalence.

(2.3) The only remaining examples of simple branched covers in the stable range are of the following form. $\varphi$ has degree $d$, and maps $M_{d k+l}$ to $N_{k}$ where $d \geq 3$ and $l>0$. We may assume that $\varphi$ is the connected sum along a singular fibre of a branched cover $\varphi_{1}$ from the disjoint union of $d-2 S^{2}$ 's, and one surface $M_{l}$ to $S^{2}$ (where $\varphi_{1}$ has degree one on each $S^{2}$ and degree 2 on $M_{l}$ ) and a branched cover $\varphi_{2}: M_{d k} \rightarrow N_{k}$ of degree $d . \varphi_{2}$ is chosen as in (2.2). See Diagram 2.3. Choose $a_{s}^{i}, b_{s}^{i}$ and $a_{s}, b_{s}$ with $\varphi_{2}\left(a_{s}^{i}\right)=a_{s}, \varphi_{2}\left(b_{s}^{i}\right)=b_{s}$ as in (2.2). Let $s_{n}, t_{n}, n=1, \ldots, l$ be the standard generators for $M_{l}$ as shown in Diagram 2.3. We have the following examples of equivalences of $\varphi$.

(2.3.1) Regard $\varphi$ as $\varphi_{1}^{\prime} \# \varphi_{2}^{\prime}$ where $\varphi_{1}^{\prime}: M_{l} \rightarrow S^{2}$ and $\varphi_{2}^{\prime}:$ ( $d$ copies of $N_{k}$ ) $\rightarrow N_{k}$. By the Realization Theorem of Berstein and Edmonds [BE 1; §4] any homeomorphism of $M_{l}$ may be realized as an equivalence of $\varphi_{1}^{\prime}$. In particular, $T\left(\left[s_{n}\right]\right), T\left(\left[t_{n}\right]\right), T\left(\left[s_{n}\right]-\left[s_{m}\right]\right), T\left(\left[t_{n}\right]-\left[t_{m}\right]\right)$ are all equivalences of $\varphi_{1}^{\prime}$ and by using Lemma (1.4) they may be extended by the identity to equivalences of $\varphi$.

(2.3.2) When $\varphi$ is regarded as $\varphi_{1} \# \varphi_{2}$, all of the equivalences of (2.2) extend to equivalences of $\varphi$. (The only change is in constructing an $\operatorname{arc} \beta$ of type (12), (12) in $N_{k}-\stackrel{\circ}{D}^{2}$ where $\stackrel{\circ}{D}^{2}$ contains a branch point of type (12).)

(2.3.3) Again regard $\varphi$ as $\varphi_{1}^{\prime} \# \varphi_{2}^{\prime}$. In the proof of Theorem (1.3), Berstein and Edmonds [BE1; §4] produce an $\operatorname{arc} \beta$ in $S^{2}$ containing * such that $\beta$ is covered by a loop homotopic to any chosen $s_{n}$. Using Lemma (1.3) we may force $\beta$ to be of type $(i j),(i j)$. If we then consider the connected sum of $\beta$ with the loop $a_{s}$ in $N_{k}$, we see that a disk twist about this arc lifts to the equivalence $T\left(\left[s_{n}\right]+\left[a_{s}^{i}\right]-\left[a_{s}^{j}\right]\right)$. Replacing $a_{s}$ by $b_{s}$ shows that $T\left(\left[s_{n}\right]+\left[b_{s}^{i}\right]-\left[b_{s}^{j}\right]\right)$ is an equivalence. Finally if we 


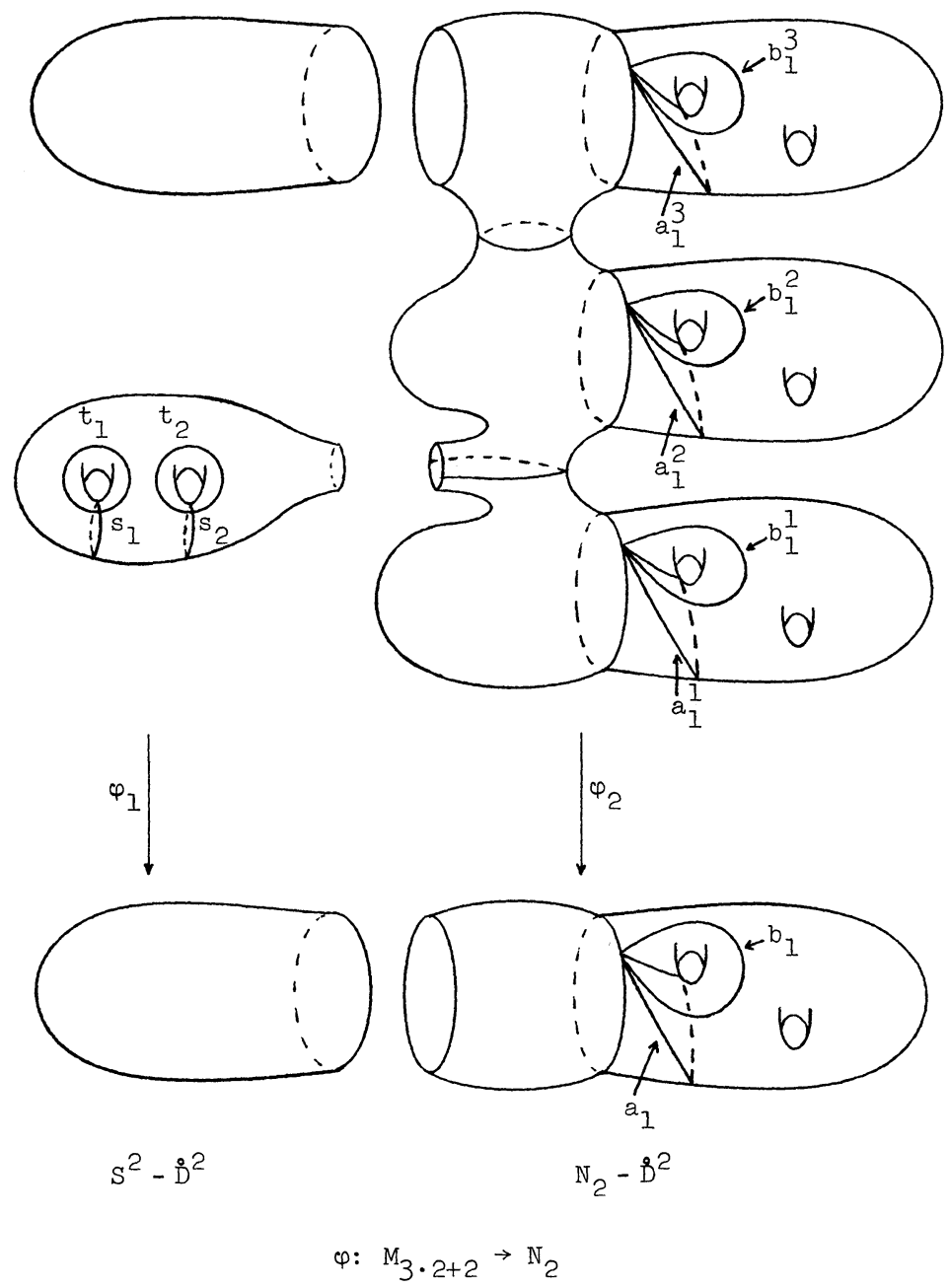

Diagram 2.3

replace $s_{n}$ by $t_{n}$ we see that $T\left(\left[t_{n}\right]+\left[a_{s}^{i}\right]-\left[a_{s}^{j}\right]\right)$ and $T\left(\left[t_{n}\right]+\left[b_{s}^{i}\right]-\left[b_{s}^{j}\right]\right)$ are equivalences.

(2.4) The most general simple branched cover was given in (2.3) (though possibly with $l=0$ ). Using the notation of $(2.3)$, we have $\varphi$ : $M_{d k+l} \rightarrow N_{k}$ where $\varphi$ may be regarded as $\varphi_{1}^{\prime} \# \varphi_{2}^{\prime}$.

(2.4.1) In [BE 1; §4], Berstein and Edmonds give ar example of an equivalence of $\varphi_{1}^{\prime}$ which reverses orientation. We may assume such an equivalence fixes a basepoint. Clearly there are basepoint fixing, orientation reversing equivalences of $\varphi_{2}^{\prime}$. Together these give an orientation reversing equivalence of $\varphi$. 
(2.4.2) In the decomposition of $\varphi$ as $\varphi_{1}^{\prime} \# \varphi_{2}^{\prime} N_{k}$ is regarded as $S^{2} \# N_{k}$ (see Diagram 2.3). Any homeomorphism of $N_{k}$ is homotopic to one fixing a neighborhood of a base point. This homeomorphism clearly lifts to an equivalence of $\varphi_{2}^{\prime}$. Taking the fibre connected sum of this equivalence with the trivial equivalence of $\varphi_{1}^{\prime}$ shows that every homeotopy class of $N_{k}$ contains a homeomorphism lifting to an equivalence of $\varphi$.

\section{CHAPTER 3}

Action of equivalences on homology and a technical lemma. In this chapter, we study the action on $H_{1}(M)$ of the equivalences of $\varphi: M \rightarrow N$ given in (2.1), (2.2) and (2.3). We also interpret right multiplication by these matrices on an arbitrary matrix $X$ as "modified column operations". Specializing to $X$ a matrix with one row we prove a technical lemma (3.8) for use in Chapter 4 that describes certain one row matrices $X^{\prime}$ to which $X$ may always be reduced.

Consider a Z-module $V$ with ordered basis $a_{1}, \ldots, a_{n}, b_{1}, \ldots, b_{n}$ and innerproduct defined by $a_{i} \cdot a_{j}=b_{i} \cdot b_{j}=0 \forall i, j, a_{i} \cdot b_{j}=b_{j} \cdot a_{i}=0$ $\forall i \neq j$, and $a_{i} \cdot b_{i}=-b_{i} \cdot a_{i}=1 \forall i$. If $X \in \mathrm{Gl}(2 n, \mathbf{Z})$, i.e. $X: V \rightarrow V$ we have 4 equivalent definitions for $X$ to be symplectic.

(1) $X$ preserves the innerproduct on $V$.

(2) The identification of $V$ with $\mathbf{Z}^{2 n}$ induces a pairing $\cdot$ on $\mathbf{Z}^{2 n}$. Under this identification

$$
(i \text { th column of } X) \cdot(n+j \text { th column of } X)=a_{i} \cdot b_{j}
$$

for all $i, j \leq n$.

(3)

$$
X^{t}\left(\begin{array}{rr}
0 & -I \\
I & 0
\end{array}\right) X=\left(\begin{array}{rr}
0 & -I \\
I & 0
\end{array}\right)
$$

where $t$ denotes transpose and $I$ is the $n \times n$ identity matrix.

(4) Replace $V$ with $H_{1}\left(M_{n}\right)$, let $\left[a_{1}\right], \ldots,\left[a_{n}\right],\left[b_{1}\right], \ldots,\left[b_{n}\right]$ be the standard generators of $H_{1}\left(M_{n}\right)$, and let $\cdot$ be the intersection pairing. The equivalent condition is $X=f_{*}$, where $f$ is a homeomorphism of $M_{n}$.

$1,2,3$ are easily seen to be equivalent, 4 is well known. The collection of all such $X$ is the symplectic group $\operatorname{Sp}(n, Z)$. It is easy to see that $X \in \operatorname{Sp}(n, \mathbf{Z})$ implies $X$ transpose $=X^{t} \in \operatorname{Sp}(n, \mathbf{Z})$.

If $c$ and $d$ are loops in $M_{n}$ then so is $T(c)(d)$, and in $H_{1}\left(M_{n}\right)$ $[T(c)(d)]=[d]+([d] \cdot[c])[c]$. In particular, if $[c]=\sum \alpha_{i}\left[a_{i}\right]+\beta_{i}\left[b_{i}\right]$ then $T(c)_{*}: H_{1}\left(M_{n}\right) \rightarrow H_{1}\left(M_{n}\right)$ is defined by

$$
\begin{gathered}
T(c)_{*}\left[a_{i}\right]=\left[a_{i}\right]+\beta_{i}[c] \text { and } \\
T(c)_{*}\left[b_{i}\right]=\left[b_{i}\right]-\alpha_{i}[c] .
\end{gathered}
$$


This shows that $T\left(c_{1}\right)_{*}=T\left(c_{2}\right)_{*}$ if $\left[c_{1}\right]=\left[c_{2}\right]$. We shall only be studying the effect of these twist homemorphisms on $H_{1}\left(M_{n}\right)$ so we shall replace the notation $T([c])$ of Chapter 2 by $T(c)$ and denote the homology classes $\left[a_{i}\right]$ and $\left[b_{i}\right]$ by $a_{i}$ and $b_{i}$. (See also [Li 1], [Li 2].)

With $c$ as above, the matrix of $T(c)_{*}$ with respect to the basis $a_{1}, \ldots, a_{n}, b_{1}, \ldots, b_{n}$ is

$$
I+\left(\begin{array}{ll}
\left(x_{i j}\right) & \left(y_{i j}\right) \\
\left(z_{i j}\right) & \left(w_{i j}\right)
\end{array}\right)
$$

where $x_{i j}=\alpha_{i} \beta_{j}, y_{i j}=-\alpha_{i} \alpha_{j}, z_{i j}=\beta_{i} \beta_{j}$ and $w_{i j}=-\alpha_{j} \beta_{i}$.

For use in Chapter 4, we now list the matrices obtained from the equivalences constructed in Chapter 2.

(3.1) As in (2.1), let $\varphi: M_{d} \rightarrow S^{1} \times S^{1}$ with $\varphi$ of degree $d$. With respect to the basis $a^{1}, \ldots, a^{d}, b^{1}, \ldots, b^{d}$ of $H_{1}\left(M_{d}\right)$

$$
\begin{aligned}
T\left(a^{i}-a^{j}\right)_{*} & =I+\left(\begin{array}{cccc}
a^{i} & a^{j} & b^{i} & b^{j} \\
0 & 0 & -1 & 1 \\
0 & 0 & 1 & -1 \\
\hline 0 & 0 & 0 & 0 \\
0 & 0 & 0 & 0
\end{array}\right), \quad i \neq j, \\
T\left(b^{i}-b^{j}\right)_{*} & =I+\left(\begin{array}{rr|cc}
0 & 0 & 0 & 0 \\
0 & 0 & 0 & 0 \\
\hline 1 & -1 & 0 & 0 \\
-1 & 1 & 0 & 0
\end{array}\right) \quad i \neq j, \\
P_{*}^{i j} & =I+\left(\begin{array}{rr|rr}
-1 & 1 & 0 & 0 \\
1 & -1 & 0 & 0 \\
\hline 0 & 0 & -1 & 1 \\
0 & 0 & 1 & -1
\end{array}\right) \quad i \neq j .
\end{aligned}
$$

In each of the above $I$ is the $2 d \times 2 d$ identity matrix and the second matrix has entries equal to 0 outside the $4 \times 4$ block corresponding to the basis elements $a^{i}, a^{j}, b^{i}, b^{j}$.

If $X$ is an arbitrary $2 d \times 2 d$ matrix, then $X \cdot T\left(a^{i}-a^{j}\right)_{*}$ is the matrix $X$ after the following modified column operation is applied. The difference of the $j$ th and $i$ th columns of $X$ are added to and subtracted from the $d+i$ th and $d+j$ th columns of $X$ respectively. Right multiplication by $T\left(b^{i}-b^{j}\right)_{*}$ performs the same modified column operation on $X$ though with the roles of $i, j$ and $d+i, d+j$ reversed. Right multiplication by $P_{*}^{j j}$ permutes the $i$ and $j$ columns and the $d+i$ and $d+j$ columns. Left multiplication by these matrices performs analogous row operations. 
(3.2) As in (2.2), let $\varphi: M_{k d} \rightarrow N_{k}$ be of degree $d$. With respect to the basis $a_{1}^{1}, \ldots, a_{1}^{d}, \ldots, a_{k}^{1}, \ldots, a_{k}^{d}, b_{1}^{1}, \ldots, b_{1}^{d}, \ldots, b_{k}^{1}, \ldots, b_{k}^{d}$ of $H_{1}\left(M_{k d}\right)$ we have

$$
\begin{aligned}
& T\left(a_{s}^{i}-a_{s}^{j}\right)_{*}=I+\left(\begin{array}{rrrrrrrr}
a_{s}^{i} & a_{s}^{j} & a_{t}^{i} & a_{t}^{j} & b_{s}^{i} & b_{s}^{j} & b_{t}^{i} & b_{t}^{j} \\
& & & & -1 & 1 & 0 & 0 \\
& & & & 1 & -1 & 0 & 0 \\
& & & & 0 & 0 & 0 & 0 \\
& & & 0 & 0 & 0 \\
\hline & 0 & & & & & & \\
& & & & &
\end{array}\right) \\
& T\left(b_{t}^{i}-b_{t}^{j}\right)_{*}=I+\left(\begin{array}{rrrr|r} 
& 0 & & 0 \\
\hline 0 & 0 & 0 & 0 & \\
0 & 0 & 0 & 0 & 0 \\
0 & 0 & 1 & -1 & \\
0 & 0 & -1 & 1 &
\end{array}\right)
\end{aligned}
$$

These matrices will be called type I.

$$
\begin{aligned}
& \therefore T\left(a_{s}^{i}-a_{s}^{j}+a_{t}^{i}-a_{t}^{j}\right)_{*} T\left(a_{s}^{i}-a_{s}^{j}\right)_{*}^{-1} T\left(a_{t}^{i}-a_{t}^{j}\right)_{*}^{-1} \\
& =I+\left(\begin{array}{rrrrrrrr}
a_{s}^{i} & a_{s}^{j} & a_{t}^{i} & a_{t}^{j} & b_{s}^{i} & b_{s}^{j} & b_{t}^{i} & b_{t}^{j} \\
& & & & 0 & 0 & -1 & 1 \\
& & & & 0 & 0 & 1 & -1 \\
& & & & -1 & 1 & 0 & 0 \\
& & & 1 & -1 & 0 & 0 \\
\hline & 0 & & & & & &
\end{array}\right)
\end{aligned}
$$

This matrix will be called type II.

$$
\begin{aligned}
& T\left(a_{s}^{i}-a_{s}^{j}+b_{t}^{i}-b_{t}^{j}\right)_{*} T\left(a_{s}^{i}-a_{s}^{j}\right)_{*}^{-1} T\left(b_{t}^{i}-b_{t}^{j}\right)_{*}^{-1} \\
&=I+\left(\begin{array}{rrrrrrrr}
a_{s}^{i} & a_{s}^{j} & a_{t}^{i} & a_{t}^{j} & b_{s}^{i} & b_{s}^{j} & b_{t}^{i} & b_{t}^{j} \\
0 & 0 & 1 & -1 & & & & \\
0 & 0 & -1 & 1 & & & & \\
0 & 0 & 0 & 0 & & & 0 & \\
0 & 0 & 0 & 0 & & & & \\
\hline & & & & 0 & 0 & 0 & 0 \\
& 0 & & & 0 & 0 & 0 \\
& & & & -1 & 0 & 0 \\
-1 & 1 & 0 & 0
\end{array}\right)
\end{aligned}
$$


This matrix will be called type III. Finally

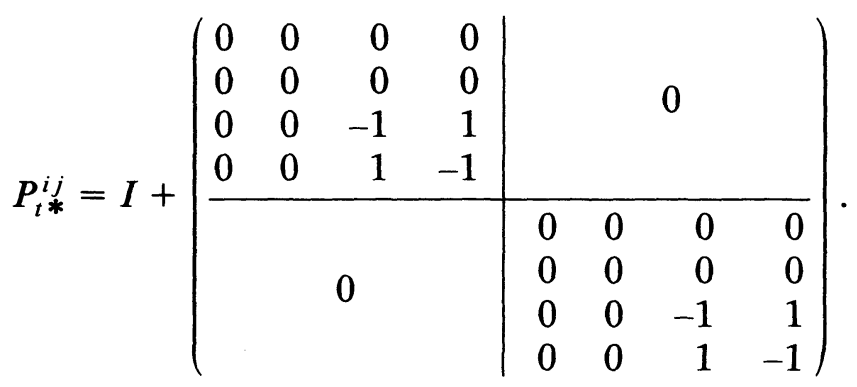

Let $X$ be an arbitrary $2 k d \times 2 k d$ matrix and consider the effect on $X$ of right multiplication by matrices of types I, II and III.

Multiplication on the right of $X$ by a type I matrix produces the same modified column operation as in the first two examples of (3.1). The only difference is that the $i$ th and $j$ th columns that are chosen in (3.1) are arbitrary while here they must correspond to basis elements with the same subscript. See Diagram 3.1.

Multiplication on the right of $X$ by a type II matrix produces a pair of the same modified column operations. That is, the difference of the $a_{t}^{i}$ and $a_{t}^{j}$ columns is added to and subtracted from the $b_{s}^{i}$ and $b_{s}^{j}$ columns while the difference of the $a_{s}^{i}$ and $a_{s}^{j}$ columns is added to and subtracted from the $b_{t}^{i}$ and $b_{t}^{j}$ columns. We can also construct, by interchanging the roles of $a$ and $b$, a matrix which allows the differences of various $b_{s}^{i}$ columns to

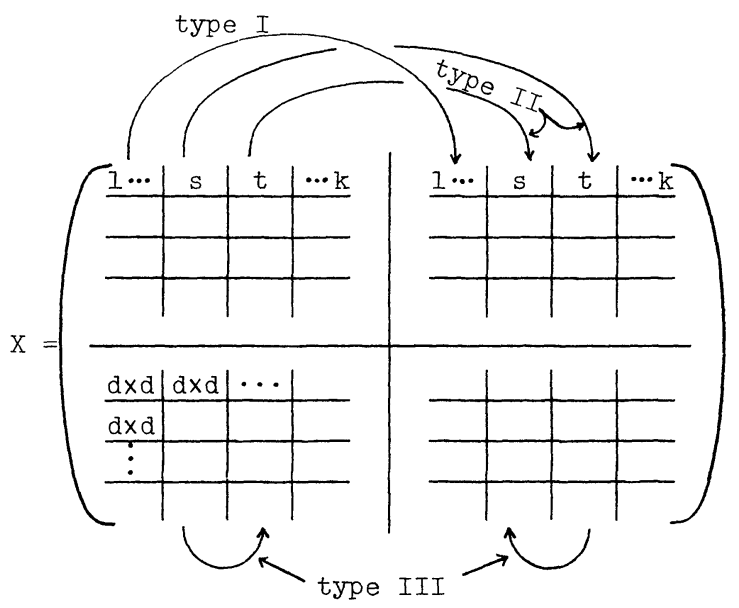

Diagram 3.1 
be added and subtracted from the appropriate $a_{s}^{i}$ columns. These will also be called type II matrices.

Multiplication on the right of $X$ by a type III matrix again produces a pair of modified column operations, though this time the difference of the $a_{s}^{i}$ and $a_{s}^{j}$ columns is added to and subtracted from the $a_{t}^{i}$ and $a_{t}^{j}$ columns while the difference of the $b_{t}^{i}$ and $b_{t}^{j}$ columns is added to and subtracted from the $b_{s}^{i}$ and $b_{s}^{j}$ columns.

Right multiplication by $P_{t *}^{i j}$ permutes two pairs of corresponding columns just as $P_{*}^{i j}$ did in (3.1), though in this case the permuted columns must have the same subscript.

Diagram 3.1 summarizes which columns the various types of matrices act on. Each long arrow represents a modified column operation as described in (3.1). The three types of operations constructed in (3.2) are combinations of modified column operations restricted to various groups of $d$ columns of $X$.

(3.3) As in (2.3), let $\varphi: M_{k d+l} \rightarrow N_{k}$ be of degree $d$. With respect to the basis $s_{1}, \ldots, s_{l}, a_{1}^{1}, \ldots, a_{1}^{d}, \ldots, a_{k}^{1}, \ldots, a_{k}^{d}, t_{1}, \ldots, t_{l}, b_{1}^{1}, \ldots$, $b_{1}^{d}, \ldots, b_{k}^{1}, \ldots, b_{k}^{d}$ we have the same equivalences with the same matrices as in example 2 and

$$
\begin{aligned}
& T\left(s_{n}\right)_{*}=I+\left(\begin{array}{cc|cc}
s_{n} & s_{m} & t_{n} & t_{m} \\
0 & 0 & -1 & 0 \\
0 & 0 & 0 & 0 \\
\hline 0 & 0 & 0 & 0 \\
0 & 0 & 0 & 0
\end{array}\right) \\
& T\left(t_{n}\right)_{*}=I+\left(\begin{array}{c|ccc}
0 & 0 & 0 & 0 \\
0 & 0 & 0 & 0 \\
\hline 1 & 0 & 0 & 0 \\
0 & 0 & 0 & 0
\end{array}\right)
\end{aligned}
$$

which we call type I. Also

$$
\begin{aligned}
& T\left(s_{n}-s_{m}\right)_{*} T\left(s_{n}\right)_{*}^{-1} T\left(s_{m}\right)_{*}^{-1}=I+\left(\begin{array}{cc|cc}
0 & 0 & 0 & 1 \\
0 & 0 & 1 & 0 \\
\hline 0 & 0 & 0 & 0 \\
0 & 0 & 0 & 0
\end{array}\right) \\
& T\left(t_{n}+t_{m}\right)_{*} T\left(t_{n}\right)_{*}^{-1} T\left(t_{m}\right)_{*}^{-1}=I+\left(\begin{array}{cc|cc}
0 & 0 & 0 & 0 \\
0 & 0 & 0 & 0 \\
\hline 0 & 1 & 0 & 0 \\
1 & 0 & 0 & 0
\end{array}\right)
\end{aligned}
$$


which we call type II. It follows that

$$
\begin{aligned}
& T\left(s_{n}+a_{s}^{i}-a_{s}^{j}\right)_{*} T\left(a_{s}^{i}-a_{s}^{j}\right)_{*}^{-1}\left(s_{n}\right)_{*}^{-1} \\
& \quad=I+\left(\begin{array}{rrrrrr}
s_{n} & a_{s}^{i} & a_{s}^{j} & t_{n} & b_{s}^{i} & b_{s}^{j} \\
& & & 0 & -1 & 1 \\
& 0 & & -1 & 0 & 0 \\
& & & 0 & 0 \\
\hline & 0 & & 0
\end{array}\right) \\
& T\left(s_{n}+b_{s}^{i}-b_{s}^{j}\right)_{*} T\left(b_{s}^{i}-b_{s}^{j}\right)_{*}^{-1} T\left(s_{n}\right)_{*}^{-1} \\
& =I+\left(\begin{array}{rrr|rrr}
0 & 1 & -1 \\
0 & 0 & 0 \\
0 & 0 & 0 & & & \\
\hline & 0 & & 0 & \\
& & & -1 & 0 & 0 \\
& & & 0 & 0
\end{array}\right) .
\end{aligned}
$$

These matrices are called types $A$ and $B$ respectively.

(3.4) We now study the effect of the modified column operations of (3.1) on a $1 \times 2 d$ matrix $X=\left(\alpha^{1}, \ldots, \alpha^{d} \mid \beta^{1}, \ldots, \beta^{d}\right)$ with entries in $\mathbf{Z}$.

Define $\Delta: \mathbf{Z}^{d} \rightarrow \mathbf{Q}$ by $\Delta(\vec{\alpha})=\sum_{i=1}^{d}\left|\alpha^{i}-a\right|$ where $\vec{\alpha}=\left(\alpha^{1}, \ldots, \alpha^{d}\right)$ and $a=(1 / d) \sum_{i=1}^{d} \boldsymbol{\alpha}^{i}$. Also denote by $\Delta$ the function $\mathbf{Z}^{d_{1}} \times \cdots \times \mathbf{Z}^{d_{k}} \rightarrow$ $\mathbf{Q}$ by $\Delta(\vec{\alpha}|\vec{\beta}| \cdots \mid \vec{\delta})=\Delta(\vec{\alpha})+\Delta(\vec{\beta})+\cdots+\Delta(\vec{\delta})$. Define $\Gamma: \mathbf{Z}^{d_{1}} \times$ $\cdots \times \mathbf{Z}^{d_{k}} \rightarrow \mathbf{Z}$ by

$$
\Gamma(\vec{\alpha}|\vec{\beta}| \cdots \mid \vec{\delta})=\operatorname{gcd}\left(\bigcup_{i=1}^{d_{k}}\left\{\boldsymbol{\alpha}^{1}-\boldsymbol{\alpha}^{i}\right\}\right) \cup \cdots \cup\left(\bigcup_{i=1}^{d_{k}}\left\{\boldsymbol{\delta}^{1}-\delta^{i}\right\}\right) .
$$

Denote by $\sim$ the equivalence relation on $\mathbf{Z}^{d} \times \mathbf{Z}^{d}$ generated by the following operations: ...).

(1) $(\vec{\alpha} \mid \vec{\beta}) \sim S_{i j}(\vec{\alpha} \mid \vec{\beta})=\left(\vec{\alpha}\left|\ldots, \beta^{i}+\right| \alpha^{i}-\alpha^{j}\left|, \ldots, \beta^{j}-\right| \alpha^{i}-\alpha^{j} \mid\right.$

(2) $(\vec{\alpha} \mid \vec{\beta}) \sim T_{i j}(\vec{\alpha} \mid \vec{\beta})=\left(\ldots, \alpha^{i}+\left|\beta^{i}-\beta^{j}\right|, \ldots, \alpha^{j}-\left|\beta^{i}-\beta^{j}\right|\right.$ $\ldots \mid \vec{\beta})$.

(3) $(\vec{\alpha} \mid \vec{\beta})=\left(\cdots \alpha^{i} \cdots \alpha^{j} \cdots \mid \cdots \beta^{i} \cdots \beta^{j} \cdots\right) \sim\left(\cdots \alpha^{j} \cdots \alpha^{i}\right.$ $\left.\cdots \mid \cdots \beta^{j} \cdots \beta^{i} \cdots\right)$ ). Notice that the first operation of (3.1) (or its inverse, depending on the sign of $\alpha^{i}-\alpha^{j}$ ) is $S_{i j}$. The second and third operations of (3.1) also have the same effect as the second and third operations here. Also notice that if $(\vec{\alpha} \mid \vec{\beta}) \sim\left(\vec{\alpha}^{\prime} \mid \vec{\beta}^{\prime}\right)$ then $\Gamma(\vec{\alpha} \mid \vec{\beta})=$ $\Gamma\left(\vec{\alpha}^{\prime} \mid \vec{\beta}^{\prime}\right)$.

We state without proof the following lemma which will be used extensively in Chapter 4. 
(3.5). LEMMA. If $\Delta$ takes on its minimum value at $(\vec{\alpha} \mid \vec{\beta})$ when restricted to the equivalence class of $(\vec{\alpha} \mid \vec{\beta})$ then $(\vec{\alpha} \mid \vec{\beta})$ must be one of the following types:

0. $\alpha^{i}=\alpha^{j}, \beta^{i}=\beta^{j} \forall i, j$.

1. (a) $\alpha^{i}=\alpha^{j} \forall i, j$ and $\#\left\{\beta^{1}, \ldots, \beta^{d}\right\}=2$.

(b) $\beta^{i}=\beta^{j} \forall i, j$ and $\#\left\{\alpha^{1}, \ldots, \alpha^{d}\right\}=2$.

II. (a) $\alpha^{i}=\alpha^{j} \forall i, j, \#\left\{\beta^{1}, \ldots, \beta^{d}\right\}=3$ and there exist exactly two extreme values while the third value is the average of the extreme values.

(b) This is the same as (a) with the roles of $\vec{\alpha}, \vec{\beta}$ reversed.

III. $\#\left\{\alpha^{1}, \ldots, \alpha^{d}\right\}=\#\left\{\beta^{1}, \ldots, \beta^{d}\right\}=2$ and $\Gamma(\vec{\alpha})=\Gamma(\vec{\beta})$.

(3.6) Corollary. Any pair $(\vec{\alpha} \mid \vec{\beta}) \in \mathbf{Z}^{d} \times \mathbf{Z}^{d}$ with $d \geq 3$ is equivalent to a pair $\left(\vec{\alpha}^{\prime} \mid \vec{\beta}^{\prime}\right)$ with $\left|\alpha^{1 \prime}-\alpha^{2 \prime}\right|=\Gamma\left(\vec{\alpha}^{\prime}\right)=\Gamma\left(\vec{\alpha}^{\prime} \mid \vec{\beta}^{\prime}\right)$.

Proof. We may assume $(\vec{\alpha} \mid \vec{\beta})$ minimizes $\Delta$. In cases 0 , IB, IIb, III of Lemma (3.5) permutations may be used to obtain the desired result. In cases Ia, IIa, $\exists i, j$ with $\left|\beta^{i}-\beta^{j}\right|=\Gamma(\vec{\alpha} \mid \vec{\beta})$. The operation $T_{i j}$ followed by a permutation taking $i$ to 1 and $j$ to 3 gives the desired $\left(\vec{\alpha}^{\prime} \mid \vec{\beta}^{\prime}\right)$.

\section{CHAPTER 4}

Proof of the Main Theorem and some open questions. Most of the chapter will be devoted to the proof of the following main result.

(4.1). THEOREM. Let $\psi_{1}, \psi_{2}$ be stable simple branched covers from $M$ to $N$, and let $f, g$ be homeomorphisms of $M$ and $N$ respectively such that $g_{*} \psi_{1 *}=\psi_{2 *} f_{*}$ from $H_{*}(M)$ to $H_{*}(N)$, then $\exists$ homeomorphisms $\bar{f}, \bar{g}$ of $M$ and $N$ such that $\bar{f}_{*}=f_{*}, \bar{g} \simeq g$, and

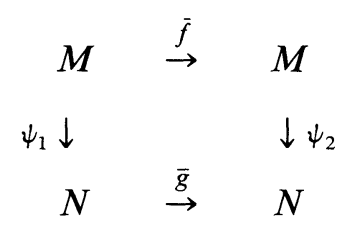

commutes.

Proof. By the Stable Uniqueness Theorem (1.2) we may assume $\psi_{1}=\psi_{2}=\varphi$ where $\varphi$ is any simple branched cover of the same degree as $\psi_{1}$ and $\psi_{2}$. The proof will split into three parts depending on the genus of $M$ and $N$ and the choice of $\varphi$. These parts are

(4.2) $\varphi: M_{d} \rightarrow N=S^{1} \times S^{1}$ as constructed in (2.1).

(4.3) $\varphi: M_{k d} \rightarrow N_{k}$ as constructed in (2.2).

(4.4) $\varphi: M_{k d+l} \rightarrow N_{k}$ as constructed in (2.3). 
The proof in each of these cases follows the same outline.

By (2.4.1) we may assume $f$ and $g$ preserve orientation, and by (2.4.2) we may assume $g \simeq \mathrm{id}$. The hypothesis that $g_{*} \psi_{1 *}=\psi_{2 *} f_{*}$ from $H_{*}(M)$ to $H_{*}(N)$ is then equivalent to $\varphi_{*}=\varphi_{*} f_{*}$ from $H_{1}(M)$ to $H_{1}(N)$.

We then study the following two groups associated with $\varphi: M \rightarrow N$. Let $E_{*}$ consist of all matrices $f_{*}$ where $f_{*}: H_{1}(M) \rightarrow H_{1}(M)$ and $f, g$ is an equivalence of $\varphi$ with $g \simeq \mathrm{id}_{N}$. Let $L$ be those symplectic matrices $A$ : $H_{1}(M) \rightarrow H_{1}(M)$ that are lifts of id: $H_{1}(N) \rightarrow H_{1}(N)$ with respect to $\varphi_{*}$, i.e., $\varphi_{*}=\varphi_{*} A$. From the hypothesis of the theorem and from the fourth definition of symplectic given in Chapter 3, we see that $E_{*} \subseteq L$. To prove the theorem, we shall show $L \subseteq E_{*}$. In (3.1), (3.2), and (3.3.), we gave examples of elements of $E_{*}$. (4.2), (4.3), and (4.4) show that these elements generate $L$ and hence $L \subseteq E_{*}$.

In (4.2), we denote $L=L(d, \mathbf{Z}) \subseteq \operatorname{Sp}(2 d, \mathbf{Z})$, in (4.3) we denote $L=L(d, k, \mathbf{Z}) \subseteq \operatorname{Sp}(2 d k, \mathbf{Z})$, and in (4.4) we denote $L=L(d, k, l, \mathbf{Z}) \subseteq$ $\mathrm{Sp}(2 d k+2 l, \mathbf{Z})$.

Let $X=\left(\begin{array}{c}A B \\ C D\end{array}\right) \in L(d, \mathbf{Z})$. Referring to (2.1), (3.1), and the specified bases of $H_{1}\left(M_{d}\right)$ and $H_{1}\left(S^{1} \times S^{1}\right)$ we see that with respect to these bases

$$
\varphi_{*}=\left(\begin{array}{cc}
\overrightarrow{1} & \overrightarrow{0} \\
\overrightarrow{0} & \overrightarrow{1}
\end{array}\right)
$$

where $\overrightarrow{1}=(1, \ldots, 1), \overrightarrow{0}=(0, \ldots, 0) \in \mathbf{Z}^{d}$. Since

$$
\left(\begin{array}{ll}
\overrightarrow{1} & \overrightarrow{0} \\
\overrightarrow{0} & \overrightarrow{1}
\end{array}\right)\left(\begin{array}{ll}
A & B \\
C & D
\end{array}\right)=\left(\begin{array}{cc}
\overrightarrow{1} & \overrightarrow{0} \\
\overrightarrow{0} & \overrightarrow{1}
\end{array}\right)
$$

we see that the sum of the elements of any column of $A$ or $D$ is 1 , while the sum of the elements of any column of $B$ or $C$ is 0 . Call this the constant column sum property. $X^{-1}$ also has the constant column sum property. Since

$$
X^{t}\left(\begin{array}{rr}
0 & -I \\
I & 0
\end{array}\right) X=\left(\begin{array}{rr}
0 & -I \\
I & 0
\end{array}\right)
$$

we see that

$$
X^{-1}=\left(\begin{array}{rr}
0 & -I \\
I & 0
\end{array}\right)^{-1} X^{t}\left(\begin{array}{rr}
0 & -I \\
I & 0
\end{array}\right)=\left(\begin{array}{rr}
0 & I \\
-I & 0
\end{array}\right)\left(\begin{array}{ll}
C^{t} & -A^{t} \\
D^{t} & -B^{t}
\end{array}\right)=\left(\begin{array}{rr}
D^{t} & -B^{t} \\
-C^{t} & A^{t}
\end{array}\right)
$$

and the constant column sum property for $X^{-1}$ implies the constant row sum property for $X$.

(4.2). THEOREM. $L(d, \mathbf{Z})$ is generated by $T\left(a^{i}-a^{j}\right)_{*}, T\left(b^{i}-b^{j}\right)_{*}$, and P* of (3.1) where $1 \leq i \leq d, 1 \leq j \leq d$ and $i \neq j$. 
Proof. We first assume $d \geq 2$. Given $X \in L(d, \mathbf{Z})$ we shall reduce it to $I$ by multiplying it on the right and the left by $T\left(a^{i}-a^{j}\right)_{*}, T\left(b^{i}-b^{j}\right)_{*}$, $P^{i j}{ }^{i j}$ and their inverses. The first step will be to use right multiplication, i.e. the modified column operations described in (3.1) to reduce the first row to $(1,0, \ldots, 0 \mid \overrightarrow{0})$.

Let $(\vec{\alpha} \mid \vec{\beta})$ be the first row of $X$. By using the first two types of column operations we may assume $\Delta(\vec{\alpha} \mid \vec{\beta})$ is minimized. We now use the results of Lemma (3.5) to see what $(\vec{\alpha} \mid \vec{\beta})$ can be.

(3.5) 0 , Ia, IIa, and IIb can't occur because $\sum_{i=1}^{d} \alpha^{i}=1$.

(3.5) Ib. Let $\delta=\Gamma(\vec{\alpha})$. Then for some $\vec{x},(\vec{\alpha} \mid \vec{\beta})=\alpha^{1}(\overrightarrow{1} \mid \overrightarrow{0})+\delta \vec{x}$. Let $\vec{y}_{1}$ and $\vec{y}_{2}$ be the $d+1$ and $d+2$ rows of $X$. Definition 2 of symplectic holds with rows replacing columns since $X \in \operatorname{Sp}(d, \mathbf{Z})$ implies $X^{t} \in \operatorname{Sp}(d, \mathbf{Z})$. $(\vec{\alpha} \mid \vec{\beta}) \cdot \vec{y}_{1}=1$ implies $\alpha^{1}+\delta \vec{x} \cdot \vec{y}_{1}=1$ (this uses the constant row sum property) therefore $\delta \mid \alpha^{1}-1 .(\vec{\alpha} \mid \vec{\beta}) \cdot \vec{y}_{2}=0$ implies $\alpha^{1}+\delta \vec{x} \cdot \vec{y}_{2}=0$, therefore $\delta \mid \alpha^{1}$. Together these show that $1=\delta=\Gamma(\vec{\alpha})$. Since $\sum_{i=1}^{d} \alpha^{i}=1$ it follows that for some $i \vec{\alpha}=\vec{e}^{i}$, the $i$ th standard basis vector of $\mathbf{Z}^{d}$. $P_{*}^{i 1}$ then forces $(\vec{\alpha} \mid \vec{\beta})=\left(\vec{e}^{1} \mid \overrightarrow{0}\right)$.

(3.5) III. Let $\delta=\Gamma(\vec{\alpha} \mid \vec{\beta})$. Then

$$
(\vec{\alpha} \mid \vec{\beta})=\alpha^{1}(\overrightarrow{1} \mid \overrightarrow{0}) \pm \delta(\vec{x} \mid \overrightarrow{0})+\beta^{1}(\overrightarrow{0} \mid \overrightarrow{1}) \pm \delta(0 \mid \vec{y})
$$

where $\vec{x}$ and $\vec{y}$ consist of only 0 's and 1's. Suppose $\vec{x}$ has $k 1$ 's and $\vec{y}$ has $l$ 1 's with $1 \leq l \leq d-1$. Since $\sum_{i=1}^{d} \alpha^{i}=1, d \alpha^{1} \pm \delta k=1$, hence $\operatorname{gcd}(d, \delta)$ $=1$. Since $\sum_{i=1}^{d} \beta^{i}=0, d \beta^{1} \pm \delta l=0$, hence $d \mid \delta l$, a contradiction since $1 \leq l \leq d-1$.

This exhausts the possibilities of (3.5) and we may assume the first row of $X$ is $\left(\vec{e}^{1} \mid \overrightarrow{0}\right)$. Since $X$ is symplectic, it follows from the row version of Definition 2 of symplectic that the $d+1$ st column of $X$ is $\left(\overrightarrow{0} \mid \vec{e}^{1}\right)$.

The next step is to reduce the first column of $X$ to $\left(\vec{e}^{1} \mid \overrightarrow{0}\right)$ without affecting the first row. By using row operations which don't effect the first row we may assume $\Delta\left(\alpha^{2}, \ldots, \alpha^{d} \mid \beta^{2}, \ldots, \beta^{d}\right)$ is minimized where $(\vec{\alpha} \mid \vec{\beta})$ is the first column of $X$ (hence $\alpha^{1}=1$ ). Again we appeal to (3.5) and look at the possible vectors $\left(\alpha^{2}, \ldots, \alpha^{d} \mid \beta^{2}, \ldots, \beta^{d}\right)$.

(3.5) Ia. Since $\sum_{i=2}^{d} \alpha^{i}=0, \alpha^{2} \cdots=\alpha^{d}=0$ and $(\vec{\alpha} \mid \vec{\beta})=\left(\vec{e}^{1} \mid \vec{\beta}\right)$. This can easily be reduced to $\left(\vec{e}^{1} \mid \overrightarrow{0}\right)$ without affecting the first row.

(3.5) Ib. Let $\Gamma(\vec{\alpha})=\delta$. Then $(\vec{\alpha} \mid \vec{\beta})=(\overrightarrow{1} \mid \overrightarrow{0})+\delta(\vec{x} \mid \overrightarrow{0})+\left(\overrightarrow{0} \mid-d \beta^{2}, \overrightarrow{0}\right)+$ $\beta^{2}(\overrightarrow{0} \mid \overrightarrow{1})$. If $\vec{y}_{2}$ is the $d+2$ nd column of $X$ then $(\vec{\alpha} \mid \vec{\beta}) \cdot y_{2}=0$ implies $1+\delta(\vec{x} \mid \overrightarrow{0}) \cdot \bar{y}_{2}+0+0=0$ (the first 0 uses the fact that the first entry of $\vec{y}_{2}$ is 0 , the second 0 follows from the constant column sum property). It follows that $\delta=1$. Since $1=\delta=\Gamma(\vec{\alpha})=\operatorname{gcd}_{i}\left\{\alpha^{1}-\alpha^{i}\right\}$ we can use row operations $T\left(a^{1}-a^{l}\right)_{*}$ to force $\beta^{1}$ to be any number, in particular $\beta^{1}=-1$. But now $\sum_{i=2}^{d} \alpha^{i}=0, \sum_{i=2}^{d} \beta^{i}=1$ and this is the same as the first 
column case except that the roles of $\vec{\alpha}$ and $\vec{\beta}$ are interchanged, $d$ is replaced by $d-1$, and the vector $\left(\alpha^{2}, \ldots, \alpha^{d} \mid \beta^{2}, \ldots, \beta^{d}\right)$ does not lie in a symplectic matrix. The symplectic matrix was used only in the case (3.5) $\mathrm{Ib}$, and in this case (note the reversal of the roles of $\vec{\alpha}$ and $\vec{\beta}$ ) $\alpha^{2}=\cdots=$ $\alpha^{d}=0$. It follows that we can always reduce $\left(\alpha^{2}, \ldots, \alpha^{d} \mid \beta^{2}, \ldots, \beta^{d}\right)$ to $\left(\overrightarrow{0} \mid \beta^{2}, \ldots, \beta^{d}\right)$, hence $(\vec{\alpha} \mid \vec{\beta})=\left(\vec{e}^{-1} \mid \vec{\beta}\right)$ which was handled above in case (3.5) Ia.

(3.5) IIa. This is similar to Ia.

(3.5) IIb. In Ib, we only used $\beta^{2}=\cdots=\beta^{d}$. This is true in this case, and the same proof may be used.

(3.5) III. As in Ib, it will be enough to show that $\Gamma(\vec{\alpha})=1$. Let $\delta=\Gamma(\vec{\alpha})$ and let $\gamma=\Gamma\left(\alpha^{2}, \ldots, \alpha^{d}\right)=\Gamma\left(\beta^{2}, \ldots, \beta^{d}\right)$. Then

$$
(\vec{\alpha} \mid \vec{\beta})=(\overrightarrow{1} \mid \overrightarrow{0})+\delta(0, \vec{x} \mid \overrightarrow{0})+\beta^{2}(\overrightarrow{0} \mid \overrightarrow{1})+\left(\overrightarrow{0} \mid \beta^{1}-\beta^{2}, \overrightarrow{0}\right)+\gamma(\overrightarrow{0} \mid 0, \vec{y}) .
$$

If $y_{2}$ is the $d+2$ column of $X$ then $(\vec{\alpha} \mid \vec{\beta}) \cdot \vec{y}_{2}=0$ implies $1+\delta(0, \vec{x} \mid \overrightarrow{0})$. $\vec{y}_{2}+0+\gamma(\overrightarrow{0} \mid 0, \vec{y}) \cdot \vec{y}_{2}=0$ which implies $\operatorname{gcd}(\gamma, \delta)=1$. Since $\exists i \geq 2$ and $j \geq 2$ with $\alpha^{i}=\alpha^{j}+\gamma$, and since $\delta \mid 1-\alpha^{i}$ and $\delta \mid 1-\alpha^{j}$ it follows that $\delta \mid \gamma . \therefore \delta=1$.

This shows that the first column of $X$ may be reduced to $\left(\vec{e}^{1} \mid \overrightarrow{0}\right)$. Since $X$ is symplectic the $d+1$ row is $\left(\overrightarrow{0} \mid \vec{e}^{1}\right)$. If we delete the first and $d+1$ rows and columns of $X$ we have a matrix in $L(d-1, \mathbf{Z})$. Induction coupled with the observation that $L(1, \mathbf{Z})$ has only one element completes the proof of Theorem (4.2).

Referring to (2.2), (3.2), and the specified bases of $H_{1}\left(M_{k d}\right)$ and $H_{1}\left(N_{k}\right)$ we see

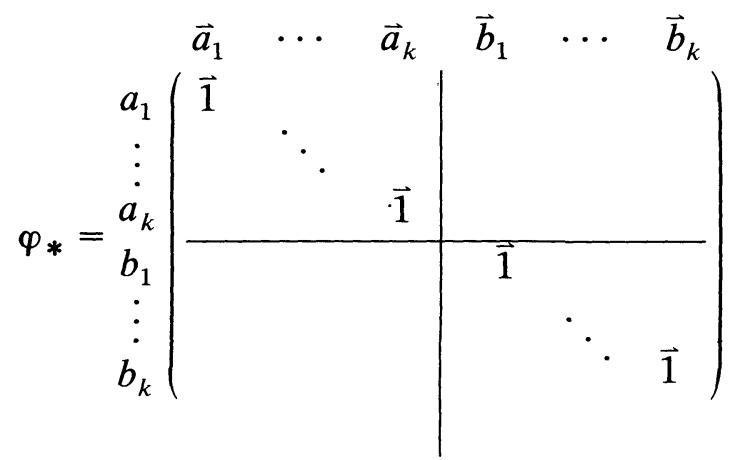

where $\vec{a}_{s}$ denotes the basis elements $a_{s}^{1}, \ldots, a_{s}^{d}, \overrightarrow{1}=(1, \ldots, 1) \in \mathbf{Z}^{d}$ and all other entries are 0 . If $X \in L(d, k, \mathbf{Z})$ then $\varphi_{*}=\varphi_{*} X$ from which it follows that $X$ has the following constant column sum property. The sum of the elements of any column in any $\vec{a}_{s} \times \vec{a}_{s}$ or $\vec{b}_{s} \times \vec{b}_{s}$ block is 1 , while the sum of elements of any column lying in a block not on the diagonal is 
0 . As before, the constant column sum property for $X^{-1}$ implies the constant row sum property for $X$.

(4.3) TheOREM. $L(d, k, \mathbf{Z})$ is generated by matrices of types I, II, and III of (3.2) together with $P_{t *}^{i j}$ where $d \geq 3, k \leq 1,1 \leq i \leq d, 1 \leq j \leq d$, $1 \leq t \leq k$ and $i \neq j$.

Proof. The case $k=1$ was proved in (4.2). We assume $k \geq 2$ and show how to reduce $k$ to $k-1$, i.e., we show how to reduce the first $d$ rows and columns of $X \in L(d, k, \mathbf{Z})$ to the first $d$ rows and columns of the identity matrix. We assume that rows and columns $1, \ldots, j$ and $d k+1, \ldots, d k+j$ of $X$ are in the desired form with $0 \leq j \leq d-1$. We first shall show how to reduce the $j+1$ row of $X$ to $\left(\vec{e}^{j+1} \mid \overrightarrow{0}\right)$ without affecting the first $j$ columns of $X$. We may assume $j \leq d-2$ for if $j=d-1$ the result is immediate from the constant column sum property.

Let $\left(\vec{\alpha}_{1}|\cdots| \vec{\alpha}_{k}|| \vec{\beta}_{1}|\cdots| \vec{\beta}_{k}\right)$ be the $j+1$ row of $X$. It follows from the inductive hypothesis that $\alpha_{1}^{i}=\beta_{1}^{i}=0$ for $i \leq j$. Let

$$
\delta=\Gamma\left(\alpha_{1}^{j+1}, \ldots, \alpha_{1}^{d}\left|\vec{\alpha}_{2}\right| \cdots\left|\vec{\alpha}_{k} \| \beta_{1}^{j+1}, \ldots, \beta_{1}^{d}\right| \vec{\beta}_{2}|\cdots| \vec{\beta}_{k}\right) .
$$

Then

$$
\begin{aligned}
\left(\vec{\alpha}_{1}|\cdots|\left|\vec{\beta}_{1}\right| \cdots\right)= & \alpha_{1}^{j+1}(\overrightarrow{1}|\overrightarrow{0}| \cdots|| \overrightarrow{0} \mid \cdots) \\
& +\alpha \vec{x}_{1}+\left({ }^{*}, \ldots,{ }^{j}, 0, \ldots, 0|\overrightarrow{0}| \cdots\right) \\
& \vdots \\
& +\alpha_{k}^{1}(\overrightarrow{0}|\cdots| \overrightarrow{1}|| \overrightarrow{0} \mid \cdots)+\delta \vec{x}_{k} \\
& +\beta_{1}^{j+1}(\overrightarrow{0}|\cdots||\overrightarrow{1}| \overrightarrow{0} \mid \cdots)+\delta \vec{y}_{1} \\
& +\left(\overrightarrow{0}\left|\cdots\|\|^{*}, \ldots,{ }^{*}, 0, \ldots, 0\right| \overrightarrow{0} \mid \cdots\right) \\
& \vdots \\
& +\beta_{k}^{1}(\overrightarrow{0}|\cdots||\overrightarrow{0}| \cdots \mid \overrightarrow{1})+\delta \vec{y}_{k} .
\end{aligned}
$$

Dotting this with the $d k+j+1$ row of $X$ shows $\delta \mid \alpha_{1}^{j+1}-1$. Dotting with the $d k+j+2$ row shows $\delta \mid \alpha_{1}^{j+1}$, therefore $\delta=1$.

By using type I column operations, we may assume $\Delta\left(\alpha_{1}^{j+1}, \ldots, \alpha_{1}^{d} \mid \beta_{1}^{j+1}, \ldots, \beta_{1}^{d}\right)$ is minimized. Since $\sum_{i=j+1}^{d} \alpha_{1}^{i}=1$ and $\sum_{i=j+1}^{d} \beta_{1}^{i}=0$ either case Ib or III of Lemma (3.5) applies, and in either case

$$
\Gamma\left(\alpha_{1}^{j+1}, \ldots, \alpha_{1}^{d}\right)=\Gamma\left(\alpha_{1}^{j+1}, \ldots, \alpha_{1}^{d} \mid \beta_{1}^{j+1}, \ldots, \beta_{1}^{d}\right)
$$


By Corollary (3.6), we may modify $\left(\vec{\alpha}_{s} \mid \vec{\beta}_{s}\right), s>1$, with type I operations so that $\Gamma\left(\vec{\alpha}_{s}\right)=\Gamma\left(\vec{\alpha}_{s} \mid \vec{\beta}_{s}\right)$. We now have $\Gamma\left(\alpha_{1}^{j+1}, \ldots, \alpha_{1}^{d}\left|\vec{\alpha}_{2}\right| \cdots \mid \vec{\alpha}_{k}\right)=1$ and this will not change under subsequent operations.

Minimize $\Delta\left(\vec{\alpha}_{2}|\cdots| \vec{\alpha}_{k}\right)$ with type III operations. It follows that $\Delta\left(\vec{\alpha}_{i} \mid \vec{\alpha}_{l}\right)$ is also minimized (with respect to type III operations involving $i$, $l \geq 2)$. By Lemma (3.8) $\Gamma\left(\vec{\alpha}_{i}\right)=\Gamma\left(\vec{\alpha}_{l}\right)$ or one of the two is 0 . It follows that $\exists i(2 \leq i \leq k)$ such that $\Gamma\left(\alpha_{1}^{j+1}, \ldots, \alpha_{1}^{d} \mid \vec{\alpha}_{i}\right)=1$. W.1.o.g. say $i=2$. This will not change under subsequent operations.

Now vary $\left(0, \ldots, 0, \alpha_{1}^{j+1}, \ldots, \alpha_{1}^{d} \mid \alpha_{2}^{1}, \ldots, \alpha_{2}^{d}\right)$ with type III operations on $\left(\alpha_{1}^{j+1}, \ldots, \alpha_{1}^{d} \mid \alpha_{2}^{j+1}, \ldots, \alpha_{2}^{d}\right)$ and permutations $P_{1 *}^{i l}(i, l \geq j+1)$ and $P_{2 *}^{i l}$ ( $i, l$ arbitrary) so that

$$
\delta=\Gamma\left(\alpha_{1}^{j+1}, \ldots, \alpha_{1}^{d} \mid \alpha_{2}^{j+1}, \ldots, \alpha_{2}^{d}\right)
$$

is minimized. By (3.5), cases Ia and IIa can't apply and we may assume (after a permutation $P_{*}^{i l} i, l \geq j+1$ ) that $\left|\alpha_{j+1}^{1}-\alpha_{j+2}^{1}\right|=\delta$. If $\delta \neq 1$, use $\Gamma\left(\alpha_{1}^{j+1}, \ldots, \alpha_{1}^{d} \mid \vec{\alpha}_{2}\right)=1$ to find $1<j+1$ such that $\delta+\alpha_{2}^{j+1}-\alpha_{2}^{i}$. The permutation $P_{2 *}^{i j+2}$ then forces

$$
\Gamma\left(\alpha_{1}^{j+1}, \ldots, \alpha_{1}^{d} \mid \alpha_{2}^{j+1}, \ldots, \alpha_{2}^{d}\right) \leq \Gamma\left(\alpha_{1}^{j+1}, \alpha_{1}^{j+2} \mid \alpha_{2}^{j+1}, \alpha_{2}^{j+2}\right)<\delta
$$

thereby contradicting the minimality of $\delta$. Therefore, we may force $\Gamma\left(\alpha_{1}^{j+1}, \ldots, \alpha_{1}^{d} \mid \alpha_{2}^{j+1}, \ldots, \alpha_{2}^{d}\right)=1$. This will not change under subsequent operations.

If we minimize $\Delta\left(\alpha_{1}^{j+1}, \ldots, \alpha_{1}^{d} \mid \alpha_{2}^{j+1}, \ldots, \alpha_{2}^{d}\right)$ with type III operations then cases Ia, IIa of (3.5) can't apply and a permutation $P_{1 *}^{j+1 l}$ forces $\vec{\alpha}_{1}=\vec{e}^{j+1}$.

Since $\vec{\alpha}_{1}=\vec{e}^{j+1}$ we can use type III operations to force $\alpha_{t}^{\prime}=0$ for $i>1, l>j=1$ without disturbing the first $j$ columns of $X$. To change $\alpha_{i}^{l}$ with $l \leq j$, use the permutation $P_{i *}^{l d}$ and then use type III operations as before. This forces $\vec{\alpha}_{2}=\cdots=\vec{\alpha}_{k}=\overrightarrow{0}$. Type I operations force $\beta_{1}^{j+1}=$ $\cdots=\beta_{1}^{d}=0$ hence $\vec{\beta}_{1}=\overrightarrow{0}$. Finally type II operations together with permutations $P_{i *}^{l d}$ are used to force $\vec{\beta}_{i}=0$ for $i \geq 2$.

The $j+1$ row is now in the desired form. Since $X$ is symplectic the $d k+j+1$ column is also in the desired form.

Let the $j+1$ column be $\left(\vec{\alpha}_{1}|\cdots| \vec{\alpha}_{k}|| \vec{\beta}_{1}|\cdots| \vec{\beta}_{k}\right)$. If $j+2=d$ then by the constant column sum property $\vec{\alpha}_{1}=\vec{e}^{j+1}$. We then proceed as before with type III and II operations together with permutations to put the entire $j+1$ column in the desired form.

Let $j+2<d$ and let

$$
\delta=\Gamma\left(\alpha_{J+1}^{1}, \ldots, \alpha_{d}^{1}\left|\vec{\alpha}_{2}\right| \cdots\left|\vec{\alpha}_{k} \| \beta_{j+2}^{1}, \ldots, \beta_{k}^{1}\right| \vec{\beta}_{2}|\cdots| \vec{\beta}_{k}\right) .
$$


Then

$$
\begin{aligned}
& \left(\vec{\alpha}_{1} \mid \cdots\right. \\
& \left.=|| \vec{\beta}_{1} \mid \cdots\right) \\
& \quad \alpha_{1}^{j+1}(\overrightarrow{1}|\overrightarrow{0}| \cdots|| \overrightarrow{0} \mid \cdots)+\delta \bar{x}_{1}+\left({ }^{1}, \ldots,{ }^{j}, 0, \ldots,|\overrightarrow{0}| \cdots|| \overrightarrow{0} \mid \cdots\right) \\
& \quad \vdots \\
& \quad+\alpha_{k}^{1}(\overrightarrow{0}|\cdots| \overrightarrow{1}|| \overrightarrow{0} \mid \cdots)+\delta \vec{x}_{k} \\
& \quad+\beta_{1}^{j+2}(\overrightarrow{0}|\cdots||\overrightarrow{1}| \overrightarrow{0} \mid \cdots)+\delta \vec{y}_{1} \\
& +\left(\overrightarrow{0}\left|\cdots \|^{*}, \ldots,^{*}, 0, \ldots, 0\right| \overrightarrow{0} \mid \cdots\right) \\
& \quad \vdots \\
& \quad+\beta_{k}^{1}(\overrightarrow{0}|\cdots||\overrightarrow{0}| \cdots \mid \overrightarrow{1})+\delta \vec{y}_{k} .
\end{aligned}
$$

Since the first $j+1$ rows and the first $j$ columns (and hence the rows $d k+1, \ldots, d k+j)$ are already in the desired form, it follows by dotting the above equation with the $d k+j+1$ and $d k+j+2$ columns that $\delta \mid \alpha_{1}^{j+1}-1$ and $\delta \mid \alpha_{1}^{j+1}$ hence $\delta=1$.

As in the case of the $j+1$ row, it will be enough to force $\vec{\alpha}_{1}=\vec{e}^{j+1}$. Towards this end minimize $\Delta\left(\alpha_{1}^{j+2}, \ldots, \alpha_{1}^{d} \mid \beta_{1}^{j+2}, \ldots, \beta_{1}^{d}\right)=\delta$ with type I operations. If $\delta=1$ it follows easily from the various caess of Lemma (3.5) (and using $\sum_{i=j+2}^{d} \alpha_{1}^{i}=0$ ) that $\vec{\alpha}_{1}$ can be reduced to $\vec{e}^{j+1}$. We now assume $\delta>1$ and show how to reduce it in each of the cases of Lemma (3.5). $\vec{e}^{j+1}$.

(3.5) Ia. This case forces $\alpha_{1}^{j+2}=\cdots=\alpha_{1}^{d}=0$, hence already $\alpha_{1}=$

(3.5) Ib. We know $\alpha_{1}^{j+1}=1$. We first suppose $\alpha_{1}^{j+1} \neq \max _{i \geq j+2} \alpha_{1}^{i}$. Pick $i \geq j+2$ such that $\alpha_{1}^{i}<0$. Then $\alpha_{1}^{j+1}-\alpha_{1}^{i}<\delta$, hence $\left(\beta_{1}^{j+1}, \ldots, \beta_{1}^{d}\right)$ can be adjusted by $\alpha_{1}^{j+1}-\alpha_{1}^{i}$ and therefore

$$
\Gamma\left(\alpha_{1}^{j+2}, \ldots, \alpha_{1}^{d} \mid \beta_{1}^{j+2}, \ldots, \beta_{1}^{d}\right) \leq \Gamma\left(\beta_{1}^{j+2}, \ldots, \beta_{1}^{d}\right) \leq \alpha_{1}^{j+1}-\alpha_{1}^{i}<\delta .
$$

Now suppose $\alpha_{1}^{j+1}=\max _{i \geq j+2} \alpha_{1}^{i}$. We've seen

$$
\Gamma\left(\alpha_{1}^{j+1}, \ldots, \alpha_{1}^{d}\left|\vec{\alpha}_{2}\right| \cdots\left|\vec{\alpha}_{k} \| \beta_{1}^{j+2}, \ldots, \beta_{1}^{d}\right| \vec{\beta}_{2}|\cdots| \vec{\beta}_{k}\right)=1
$$

By Corollary (3.6), we may force $\Gamma\left(\vec{\alpha}_{s}\right)=\Gamma\left(\vec{\alpha}_{s} \mid \vec{\beta}_{s}\right)$ for $s \geq 2$. Using (3.6) with induction, we may force $\left|\alpha_{2}^{j+1}-\alpha_{2}^{j+2}\right|=\Gamma\left(\vec{\alpha}_{2}\right)=\Gamma\left(\vec{\alpha}_{2}|\cdots| \vec{\alpha}_{d}\right)$. Therefore

$$
\Gamma\left(\alpha_{1}^{j+1}, \ldots, \alpha_{1}^{d}\left|\alpha_{2}^{j+1}, \alpha_{2}^{j+2}\right| \beta_{1}^{j+2}, \ldots, \beta_{1}^{d}\right)=1
$$


Now adjust $\left(\beta_{1}^{j+1}, \ldots, \beta_{1}^{d}\right)$ with a type II operation by $\alpha_{2}^{j+1}-\alpha_{2}^{j+2}$ and we have

$$
\Gamma\left(\alpha_{1}^{j+1}, \ldots, \alpha_{1}^{d} \mid \beta_{1}^{j+2}, \ldots, \beta_{1}^{d}\right)=1 .
$$

Since $\alpha_{1}^{j+1}=\max _{i \geq J+2} \alpha_{1}^{l}$ it follows that

$$
\Gamma\left(\alpha_{1}^{j+2}, \ldots, \alpha_{1}^{d} \mid \beta_{1}^{j+2}, \ldots, \beta_{1}^{d}\right)=1 .
$$

(3.5) IIb. In this case $\exists i \geq j+2$ with $\alpha_{1}^{i}=0$. Adjusting $\left(\beta_{1}^{j+1}, \ldots, \beta_{1}^{d}\right)$ by $\alpha_{1}^{j+1}-\alpha_{1}^{i}=1$ forces $\Gamma\left(\beta_{1}^{j+2}, \ldots, \beta_{1}^{d}\right)=1$, hence

$$
\Gamma\left(\alpha_{1}^{j+2}, \ldots, \alpha_{1}^{d} \mid \beta_{1}^{j+2}, \ldots, \beta_{1}^{d}\right)=1 .
$$

(3.5) III. The proof given in (3.5) Ib works in this case also.

In all cases the $j+1$ column and hence the $d k+j+1$ row may be reduced to the desired form. This completes the proof of Theorem (4.3).

Referring to (2.3), (3.3), and the specified bases of $H_{1}\left(M_{k d+l}\right)$ and $H_{1}\left(N_{k}\right)$ we see

$$
\left.\varphi_{*}=\begin{array}{c|cccc|cccc}
a_{1} & \vec{s} & \vec{a}_{1} & \cdots & \vec{a}_{k} & \vec{t} & \vec{b}_{1} & \cdots & \vec{b}_{k} \\
\vdots & \overrightarrow{1} & & & \overrightarrow{0} & & & \\
a_{k} & \overrightarrow{\overrightarrow{0}} & & & \overrightarrow{1} & \vdots & & & \\
b_{k} & \overrightarrow{0} & & & & \overrightarrow{0} & & & \\
\vdots & \vdots & & & & \vdots & \ddots & \\
b_{k} & \overrightarrow{0} & & & & \overrightarrow{0} & & & \overrightarrow{1}
\end{array}\right)
$$

where $\vec{s}$ denotes the basis elements $s_{1}, \ldots, s_{l}$ and $\vec{a}_{s}$ denotes the basis elements $a_{s}^{1}, \ldots, a_{s}^{d}$. If $X \in L(d, k, l, \mathbf{Z})$ then $\varphi_{*}=\varphi_{*} X$ from which it follows that $X$ must have the following constant column sum property. The sum of the elements of any column in any $\vec{a}_{s} \times \vec{a}_{s}$ or $\vec{b}_{s} \times \vec{b}_{s}$ block is 1 . The sum of the elements of any column in any $\vec{a}_{s} \times \vec{a}_{t}(s \neq t), \vec{b}_{s} \times \vec{b}_{t}$ $(s \neq t), \vec{a}_{s} \times \vec{b}_{t}$ (any $\left.s, t\right)$, or $\vec{b}_{s} \times \vec{a}_{t}$ (any $\left.s, t\right)$ block is 0 . Also, the sum of the elements of any column of the $\vec{s} \times \vec{a}_{t}, \vec{s} \times \vec{b}_{t}, \vec{t} \times \vec{a}_{s}$, and $\vec{t} \times \vec{b}_{s}$ blocks is 0 . As before, the constant column sum property for $X^{-1}$ implies a constant row sum property for $X$. The row sum agrees with the column sum in the $(\vec{a}$ or $\vec{b}) \times(\vec{a}$ or $\vec{b})$ blocks, but it applies only to the $\vec{a}_{t} \times \vec{s}$, $\vec{a}_{s} \times \vec{t}, \vec{b}_{s} \times \vec{t}$, and $\vec{b}_{t} \times \vec{s}$ blocks.

(4.4). TheOREM. $L(d, k, l, \mathbf{Z})$ is generated by matrices of types I, II, A and $\mathrm{B}$ of (3.3) together with the matrices listed in (4.3). Here $d \geq 3, k \geq 1$, $l \geq 0$. 
Proof. The proof will be by induction on $l$. The case $l=0$ is Theorem (4.3). Let $X \in L(d, k, l, \mathbf{Z})$, and let the first row of $X$ be

$$
\vec{v}=\left(\sigma_{1}, \ldots, \sigma_{l}\left|\vec{\alpha}_{1}\right| \cdots\left|\vec{\alpha}_{k} \| \tau_{1}, \ldots, \tau_{l}\right| \vec{\beta}_{1}|\cdots| \vec{\beta}_{k}\right) .
$$

We first show how to reduce $\vec{v}$ to $\left(\vec{e}^{1}|\overrightarrow{0}| \cdots|| \overrightarrow{0} \mid \cdots\right)$. Let $\delta=$ $\operatorname{gcd}\left\{\sigma_{1}, \ldots, \sigma_{l}, \alpha_{s}^{1}-\alpha_{s}^{i}, \tau_{1}, \ldots, \tau_{l}, \beta_{t}^{1}-\beta_{t}^{j}\right\}_{i, j, s, t}$, i.e.

$$
\delta=\Gamma\left(\sigma_{1}, \vec{\sigma}\left|\vec{\alpha}_{1}\right| \cdots\left|\vec{\alpha}_{k}\right|\left|\tau_{1}, \vec{\tau}\right| \vec{\beta}_{1}|\cdots| \vec{\beta}_{k}\right) .
$$

It follows that

$$
\begin{aligned}
\vec{v}= & \delta \vec{x}+\alpha_{1}^{1}(\overrightarrow{0}|\overrightarrow{1}| \overrightarrow{0}|\cdots||\overrightarrow{0}| \cdots)+\delta \vec{x}_{1} \\
& \vdots \\
& +\alpha_{k}^{1}(\overrightarrow{0}|\cdots| \overrightarrow{1}|| \overrightarrow{0} \mid \cdots)+\delta \vec{x}_{k} \\
& +\delta \vec{y}+\beta_{1}^{1}(\overrightarrow{0}|\cdots||\overrightarrow{0}| \overrightarrow{1}|\overrightarrow{0}| \cdots)+\delta \vec{y}_{1} \\
& \vdots \\
& +\beta_{k}^{1}(\overrightarrow{0}|\cdots||\overrightarrow{0}| \cdots \mid \overrightarrow{1})+\delta \vec{y}_{k} .
\end{aligned}
$$

Let $\vec{z}_{t}$ be the $l+d k+l+(t-1) d+1$ row of $X . \vec{v} \cdot \vec{z}_{t}=0$ implies

$$
\delta\left(\vec{x}+\sum_{s=1}^{k} \vec{x}_{s}+\vec{y}+\sum_{s=1}^{k} \vec{y}_{s}\right) \cdot z_{t}+\alpha_{t}^{1}=0 .
$$

Therefore $\delta \mid \alpha_{t}^{1}$ and hence $\delta \mid \alpha_{t}^{i}$ for all $i$ and $t$. By dotting $\vec{v}$ with rows in the upper half of $X$, we see $\delta \mid \beta_{t}^{1}$ and hence $\delta \mid \beta_{t}^{i}$ for all $i$ and $t$. It follows that $\delta \mid \vec{v}$, since $X$ is invertible $\delta=1$.

Notice that if $\vec{\sigma}=\vec{\tau}=\overrightarrow{0}$ there must exist $i, j, s$ such that either $\alpha_{s}^{i} \neq \alpha_{s}^{j}$ or $\beta_{s}^{i} \neq \beta_{s}^{j}$. A type $A$ or $B$ column operation forces $\vec{\tau} \neq \overrightarrow{0}$.

We now use type I operations (this includes their inverses) to simplify $(\vec{\sigma} \mid \vec{\tau})$. With these operations it is easy to see that each pair $\left(\sigma_{n} \mid \tau_{n}\right)$ may be reduced to $\left(\delta_{n} \mid 0\right)$ with $\delta_{n}=\operatorname{gcd}\left(\sigma_{n}, \tau_{n}\right)(\operatorname{see}[\mathrm{BE} 1 ;(5.4)])$, and hence $(\vec{\sigma} \mid \vec{\tau})$ may be reduce to $\left(\delta_{1}, \ldots, \delta_{l} \mid 0, \ldots, 0\right) .\left(\delta_{1}, \delta_{2} \mid 0,0\right)$ may be changed to $\left(\delta_{1}, \delta_{2} \mid \delta_{2}, \delta_{1}\right)$ by a type II operation, and this may be changed to $\left(\delta_{1,2}, 0 \mid 0, \delta_{1,2}\right)$ (where $\left.\delta_{1,2}=\operatorname{gcd}\left(\delta_{1}, \delta_{2}\right)\right)$ by type I operations. A type II operation changes this to $\left(\delta_{1,2}, 0 \mid 0,0\right)$. Continuing with $\left(\delta_{1,2}, \delta_{3} \mid 0,0\right)$ we eventually reduce $\left(\sigma_{1}, \ldots, \sigma_{l} \mid \tau_{1}, \ldots, \tau_{l}\right)$ to $\left(\delta_{\sigma, \tau}, 0, \ldots, 0 \mid 0, \ldots, 0\right)$ where $\delta_{\sigma, \tau}$ $=\operatorname{gcd}\left\{\sigma_{1}, \ldots, \sigma_{l}, \tau_{1}, \ldots, \tau_{l}\right\}$.

We next show that we may assume $\delta_{\sigma, \tau}=1$. Consider all possible first rows obtainable from $v$ using operations of types I, II, A, and B. Choose 
such a row in which $\left(\sigma_{1}, \ldots, \sigma_{l} \mid \tau_{1}, \ldots, \tau_{l}\right)=(\delta, 0, \ldots, 0 \mid 0, \ldots, 0)$ and $\delta>0$ is the minimum possible. (The last paragraph shows that there are such rows.) We now show $\delta=1$. If we let $\delta_{\alpha}=\Gamma\left(\vec{\alpha}_{1}|\cdots| \vec{\alpha}_{k}\right)$ and $\delta_{\beta}$ $=\Gamma\left(\vec{\beta}_{1}|\cdots| \vec{\beta}_{k}\right)$ then it is enough to show $\delta \mid \delta_{\alpha}$ and $\delta \mid \delta_{\beta}$, for we've seen $\operatorname{gcd}\left\{\delta, \delta_{\alpha}, \delta_{\beta}\right\}=1$. By using operations of type $A$ we can force $(\delta, 0, \cdots \mid 0, \cdots)$ to become $\left(\delta, 0 \cdots \mid \delta_{\alpha}, 0, \cdots\right)$. By the last paragraph and minimality of $\delta$ we see that $\delta \mid \delta_{\alpha}$. Similarly $\delta \mid \delta_{\beta}$, therefore $\delta=1$ and $(\vec{\sigma} \mid \vec{\tau})=\left(\vec{e}^{1} \mid \overrightarrow{0}\right)$.

Operations of type $A$ and the fact that $\sum_{i=1}^{d} \beta_{s}^{l}=0$ may be used to force $\vec{\beta}_{s}=\overrightarrow{0}$ for all $s$. Similarly, operations of type $B$ force $\vec{\alpha}_{s}=\overrightarrow{0}$ for all $s$. Operations of type $A$ and $B$ do effect $\vec{\tau}$, but $\vec{\tau}$ may be restored to $\overrightarrow{0}$ as above with type I and II operations. The first row is now in the desired form $\left(\vec{e}^{1}|\overrightarrow{0}| \cdots|| \overrightarrow{0} \mid \cdots\right)$.

Let $\left(\vec{\sigma}\left|\vec{\alpha}_{1}\right| \cdots\left|\vec{\alpha}_{k} \| \vec{\tau}\right| \vec{\beta}_{1}|\cdots| \vec{\beta}_{k}\right)$ be the first column of $X$. We've already forced $\sigma_{1}=1$. By using type I row operations, we may assume $(\vec{\sigma} \mid \vec{\tau})=\left(1, \sigma_{2}, \ldots, \sigma_{l} \mid 0, \tau_{2}, \ldots, \tau_{l}\right)$. With type I and II row operations not changing the first row of $X$, this may be reduced to $(1,0, \ldots, 0 \mid 0, \delta, 0, \ldots, 0)$. Type II operations reduce this to $\left(\vec{e}^{1} \mid \overrightarrow{0}\right)$.

Exactly as for the first row, type $\mathrm{A}$ and $\mathrm{B}$ operations allow us to reduce the entire column to $\left(\vec{e}^{1}|\overrightarrow{0}| \cdots|| \overrightarrow{0} \mid \cdots\right)$. Since $X$ is symplectic the $l+d k+1$ row and column are also in the desired form. This reduces us from $X \in L(d, k, l, \mathbf{Z})$ to $X \in L(d, k, l-1, \mathbf{Z})$ thereby completing the inductive step and the proof of Theorem (4.4).

(4.5) We now mention two open questions along the lines of Theorem (4.1).

(1) Is Theorem (4.1) true if the simple branched covers $\psi_{1}$ and $\psi_{2}$ are assumed to be metastable but not stable?

The uniqueness theorem (1.1) applies to the metastable range so one may construct self equivalences of some fixed metastable branched cover $\varphi: M \rightarrow N$ and then ask if applying $H_{*}$ to these equivalences generates $L=\left\{f_{*} \mid f\right.$ is a homeomorphism of $M$ and $\left.\varphi_{*} f_{*}=\varphi_{*}\right\}$. The problem is the complexity of these groups $L$. For example if $\varphi: M_{2} \rightarrow S^{1} \times S^{1}$ is of degree 3 then $L$ is isomorphic to the kernel $G_{3}$ of the map induced by projection $\mathbf{Z} \rightarrow \mathbf{Z} / 3 \mathbf{Z}$ in the sequence $1 \rightarrow G_{3} \rightarrow \operatorname{Sl}(2, \mathbf{Z}) \rightarrow \operatorname{Sl}(2, \mathbf{Z} / 3 \mathbf{Z})$ $\rightarrow 1$.

(2) Is Theorem (4.1) true if homology is replaced by homotopy. That is, if $\psi_{1}$ and $\psi_{2}$ are stable simple branched covers from $M$ to $N$, and $f, g$ are homeomorphisms of $M, N$ respectively such that $g \psi_{1} \simeq \psi_{2} f$ do there 
exists homeomorphisms $\bar{f}, \bar{g}$ with $\bar{f} \simeq f, \bar{g} \simeq g$ such that

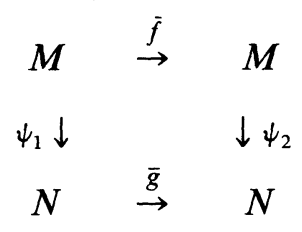

commutes?

By work of Edmonds [Ed; Cor. 4.2], the homotopy $h: M \times I \rightarrow N \times I$ between $g \psi_{1}$ and $\psi_{2} f$ may be replaced (rel $M \times\{0\} \cup M \times\{1\}$ ) by a simple branched cover (though no longer a level preserving homotopy) $\bar{H}$ : $M \times I \rightarrow N \times I$. To prove (2) it is enough to either modify $\bar{H}$ so that it becomes a level preserving simple branched cover, or equivalently to modify $\bar{H}$ so that its branch set is a braid in $N \times I$, i.e. a disjoint collection of arcs in $N \times I$ monotone with respect to the coordinate $I$.

\section{REFERENCES}

[BE 1] I. Berstein and A. Edmonds, On the construction of branched coverings of low dimensional manifolds, Trans. Amer. Math. Soc., 247 (1979), 87-124.

[BE 2] _ On the classification of generic branched coverings of surfaces, to appear in Illinois J. Math.

[Bir] J. Birman, Braids, Links, and Mapping Class Groups, Ann. of Math. Study No. 82, Princeton University Press, 1960.

[Ed] A. Edmonds, Deformation of maps to branched coverings in dimension three, Math. Ann., 245 (1979), 273-279.

[Ha] R. Hamilton, Variation of Structure on Riemann Surfaces, Princeton University Thesis, 1966.

[Hil] H. M. Hilden, Three-fold branched coverings of $S^{3}$, Amer. J. Math., 98 (1976), 989-997.

[Hir] U. Hirsch, Offene Abbildungen von Flächen auf die 2-Sphäre mit mimimalen Defekt, Arch. Math., 27 (1976), 649-656.

[Hu] A. Hurwitz, Uber Riemann'sche Flächen mit gegebenen Verzweigungspunkten, Math. Ann., 39 (1891), 1-60.

[Li 1] W. B. R. Lickorish, Homeomorphisms of non-orientable two manifolds, Proc. Camb. Phil. Soc., 59 (1963), 307-317.

[Li 2] _ A finite set of generators for the homeotopy group of a 2-manifold, Proc. Camb. Phil. Soc., 60 (1964), 769-778. Corrigendum: Proc. Camb. Phil. Soc., 62 (1966), 679-681.

[Lu] J. Lüroth, Note Über Verzweigungsschnitte und Querschnitte in einer Riemann'schen Fläche, Math. Ann., 4 (1891), 181-184.

Received December 28, 1983. Research partially supported by NSF grant DMS84-04535. 



\title{
PACIFIC JOURNAL OF MATHEMATICS EDITORS
}

DONALD BABBITT (Managing Editor)
University of California
Los Angeles, CA 90024
CHARLEs R. DEPrIMA
California Institute of Technology
Pasadena, CA 91125
R. FINN
Stanford University
Stanford, CA 94305

DoNAld BABBitT (Managing Editor)

Hermann FlaschKa

University of Arizona

Tucson, AZ 85721

RAmesh A. Gangolli

University of Washington

Seattle, WA 98195

ROBION KIRBY

University of California

Berkeley, CA 94720

C. C. MOORE

University of California

Berkeley, CA 94720

\author{
Hugo Rossi \\ University of Utah \\ Salt Lake City, UT 84112 \\ H. SAMELSON \\ Stanford University \\ Stanford, CA 94305 \\ HAROLD STARK \\ University of California, San Diego \\ La Jolla, CA 92093
}

ASSOCIATE EDITORS

R. ARENS

E. F. BECKENBACH
(1906-1982)

B. H. NEUMANN

F. WOLF

K. Yoshida

\section{SUPPORTING INSTITUTIONS}

UNIVERSITY OF ARIZONA

UNIVERSITY OF BRITISH COLUMBIA

CALIFORNIA INSTITUTE OF TECHNOLOGY

UNIVERSITY OF CALIFORNIA

MONTANA STATE UNIVERSITY

UNIVERSITY OF NEVADA, RENO

NEW MEXICO STATE UNIVERSITY

OREGON STATE UNIVERSITY
UNIVERSITY OF OREGON

UNIVERSITY OF SOUTHERN CALIFORNIA

STANFORD UNIVERSITY

UNIVERSITY OF HAWAII

UNIVERSITY OF TOKYO

UNIVERSITY OF UTAH

WASHINGTON STATE UNIVERSITY

UNIVERSITY OF WASHINGTON 


\section{Pacific Journal of Mathematics}

\section{Vol. 118, No. $1 \quad$ March, 1985}

Dan Amir, On Jung's constant and related constants in normed linear spaces ...1 Abdul Aziz, On the location of the zeros of certain composite polynomials . . 17 Joseph Barback, On hereditarily odd-even isols and a comparability of

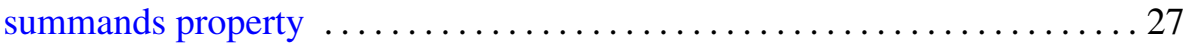

Matthew G. Brin, Klaus Johannson and Peter Scott, Totally peripheral 3-manifolds ........................................ 37

Robert F. Brown, A topological bound on the number of distinct zeros of an

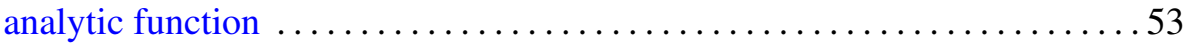

K. C. Chattopadhyay, Not every Lodato proximity is covered .......... 59

Beverly Diamond, Some properties of almost rimcompact spaces . .......63 63

Manfred Dugas and Rüdiger Göbel, On radicals and products . ......... 79

Abdelouahab El Kohen, A hyperbolic problem .................. 105

Harry Gonshor, Remarks on the Dedekind completion of a nonstandard model of the reals ................................... 117

William H. Kazez, On equivalences of branched coverings and their action

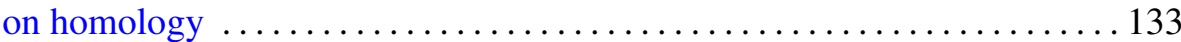

Darrell Conley Kent, On the Wallman order compactification .......... 159

Martin Andrew Magid, Lorentzian isoparametric hypersurfaces . . . . . . 165

Milan Miklavčič, Stability for semilinear parabolic equations with noninvertible linear operator

Richard Dean Neidinger and Haskell Paul Rosenthal, Norm-attainment of linear functionals on subspaces and characterizations of Tauberian operators

Johannes Vermeer, Closed subspaces of $H$-closed spaces 